\title{
Singlet particle extension of the minimal Higgs sector
}

\author{
Sissi Kyriazidou ${ }^{1}$ \\ Randall Laboratory of Physics, University of Michigan, Ann Arbor, MI 48109, USA \\ Received 28 October 1992 \\ Accepted for publication 30 November 1992
}

\begin{abstract}
A scalar singlet particle, named $U$, is coupled to the Higgs sector of the standard model for the purpose of parametrizing the large Higgs mass behaviour of the theory. The radiative corrections to the low-energy parameters ( $\rho$ parameter, $W$ mass shift) are computed at one- and two-loop levels. The new contributions demonstrate the arbitrariness of the heavy-Higgs theory, i.e., the dependence on the way of taking the large-Higgs-mass limit, but they fail to restore its uniqueness. The $\mathrm{U}$ particle effects at high energies are examined in accordance with partial wave analysis methods developed in the study of $\pi \pi$ scattering. The corrections to the $\beta$ parameter are derived from the longitudinal vector boson scattering amplitude calculated below the $1 \mathrm{TeV}$ threshold. Assuming a small, of the order of $10 \%$, difference between the masses of the scalars, we find a small but positive enhancement to the standard model contributions. The overall result indicates that no resonance will appear in the isospin $I=1$ channel at any energy above the threshold.
\end{abstract}

\section{Introduction}

The least understood part of the standard model is the Higgs sector which provides the mass generation for the various fields by means of their interaction with the Higgs scalar. The theoretical role of the Higgs sector is that it renders the theory renormalizable. In this sense the Higgs field functions as an ultraviolet regulator [1]. Thus one would expect certain radiative corrections to grow with growing Higgs mass. However, at low energies (below $100 \mathrm{GeV}$ ) the Higgs contributions to radiative corrections are at most logarithmic at the one-loop level. Due to this "screening theorem" [1] no evidence for the scalar sector has been found so far. The main goal of the next generation of colliders is to test the standard model at higher energies above the screening. The outcome is expected to follow one of two distinct possibilities [1-3]: Either a light Higgs particle (with mass much lower than $1 \mathrm{TeV}$ ) will be discovered or the weak interactions become strong at high energies. According to unitarity considerations or arguments relative

\footnotetext{
1 Present address: Physics Department, Brookhaven National Laboratory, Upton, NY 11973, USA.
} 
to the size of radiative corrections, the threshold of the new phenomena is expected at around $1 \mathrm{TeV}$.

We examine aspects of this latter possibility, namely, the heavy-Higgs-mass theory. In this perspective, the Higgs sector may be considered as an effective low-energy representation of the unknown high-energy structure, given that it provides the renormalizability of the model. Accordingly, the mass of the Higgs becomes the natural cutoff of the theory and by taking it to be infinitely large $(m \rightarrow \infty)$ the particle itself is removed and we may study the cutoff sensitivity [1]. However, previous studies revealed that the heavy-Higgs theory is not well defined. The limit $m \rightarrow \infty$ may be taken at the tree level (non-linear $\sigma$-model) or, the scalar sector of the standard model may be studied as a function of a very large, ultimately infinite, Higgs mass (linear $\sigma$-model). While at the one-loop level the limit is unique in the sense that there exists a correspondence between the coefficients of the single poles in the former and of the logarithmic $\left(\ln \left(m^{2}\right)\right)$ terms in the latter [4], this correspondence is lost at the two-loop level. An extension of this analogy at the two-loop level would require a correspondence between the quadratic divergences ( $n=3$ poles, where $n$ is the dimensionality of space-time) of the non-linear $\sigma$-model and the quadratic terms in the Higgs mass of the linear $\sigma$-model. Explicit calculation of radiative corrections $[5,6]$ at the two-loop level with the latter reveals the existence of $m^{2}$ effects that have no analogue in the corresponding result of the non-linear $\sigma$-model. Due to the appearance of these ambiguous terms, the uniqueness of the theory in the limit breaks down at the two-loop level. On the other hand, it may be that there exists a more complicated correspondence between the two models at the two-loop level, but nothing more is known at this moment.

The arbitrariness of the heavy-Higgs-mass theory may be demonstrated by means of a scalar and singlet particle, named $\mathrm{U}$, coupled exclusively to the Higgs sector of the standard model [7]. The $U$ particle affects the limit in the following sense: A heavy $U$ coupled strongly only to the Higgs sector is invisible at low energies. The appearance of its contributions in radiative corrections designates the effects that are ambiguous in the limit. A such, $U$ is a technical lever to parametrize the large-Higgs-mass effects. In other words, heavy objects interacting only with the Higgs do not seem to decouple, despite the fact that the minimal Higgs is enough to restore renormalizability. In this respect, it may be quite interesting to examine what the possible structures that affect the large-Higgs-mass limit are, how they affect precisely the observable quantities and finally whether they really represent anything about physics above the threshold. Thus, in view of the arbitrariness, it appears necessary to follow the only alternative route and to consider the consequences of a variety of heavy-Higgs sectors. It is worth noting here that the experimentally verified value for the $\rho$ parameter $(\rho=1)$ suggests that the more likely candidates for extensions of the minimal Higgs sector are singlet particles, since other representations are either forbidden or seem unnatu- 
ral in the sense that they require a fine tuning to ascertain the masslessness of the photon $[8,9]$.

In this paper we investigate a general gauge invariant coupling of a singlet particle to the minimal Higgs sector which generalizes the coupling of ref. [7]. Two models emerge, models A, B depending on whether $U$ develops a vacuum expectation value or not. This work may be divided into two parts: The first part examines the U-particle radiative corrections to low-energy parameters such as the $\rho$ parameter and the gauge boson mass shifts. In the case of a heavy $U$ field, the appearance of $m^{2}$ corrections will demonstrate the role of $\mathrm{U}$ as a means of parametrizing the ambiguity in the heavy-Higgs-mass limit. Moreover, the new contributions may be such as to cancel the aforementioned ambiguous terms that appear in the linear $\sigma$-model. It is possible that an extra structure in the scalar sector is responsible for the apparent ambiguity. A model that would succeed in this would gain a lot of credibility and would require further investigations.

The second part of the paper is involved with calculating the U-particle effects upon the longitudinal WW scattering at center of mass energies $M^{2} \ll s \ll m^{2}$ ( $M$ being the vector boson mass). At energies below the threshold at $1 \mathrm{TeV}$ perturbative calculations are still a valid approximation in the sense that the one-loop corrections due to a heavy Higgs mass are within $10 \%[7,10]$. The question is how to make definite predictions for energies higher than $1 \mathrm{TeV}$ where the structure of the Higgs sector, in case it is strongly interacting, will become apparent. It has been proposed $[3,11,12]$ that the analogy between low-energy $\pi \pi$ scattering and the tree-level longitudinal WW scattering may suggest the existence of a resonance in the isospin $I=1$ channel for the latter at energies above $1 \mathrm{TeV}$. This similarity is established in terms of the equivalence theorem $[2,3,13]$ which relates the unphysical Higgs $\phi \phi$ amplitude to the $\mathrm{W}_{\mathrm{L}} \mathrm{W}_{\mathrm{L}}$ amplitude at energies higher than the mass of the vector bosons and it is based on the fact that both the pion and the Higgs systems are described by the $\sigma$-model. Recently it was shown [14] that the position of the resonance may be specified in terms of the Lehmann $\beta$ parameter and its value may be derived from the one-loop amplitude of $\mathrm{W}_{\mathrm{L}} \mathrm{W}_{\mathrm{L}}$ at energies below $1 \mathrm{TeV}$. The treatment parallels the discussion of the pion system by Lehmann [15] and the validity of the approach is based on its successes in pion physics. Thus, when $\mathrm{W}_{\mathrm{L}} \mathrm{W}_{\mathrm{L}}$ scattering is observed in the future colliders, the parameter $\beta$ will be measured. The various models presently considered will be tested according to the value they predict for this parameter. In this sense, $\beta$ is a parameter of great phenomenological significance. However, within the context of the heavy-Higgs theory, its value is arbitrary. The calculation of $\beta$ in the largeHiggs-mass limit indicates once again a dependence on the way of taking the limit and the arbitrariness may be demonstrated in terms of the U-particle effects $[14,16]$. Given that the large-Higgs-mass limit is not well defined but it allows for an additional structure in the scalar sector, we will examine how the $\beta$-parameter is affected by our models for a singlet particle $U$. 
The paper is organized as follows: In sect. 2 two models for the U particle are derived, starting from a gauge invariant coupling of a singlet to the minimal Higgs sector. In sect. 3 their effects to the low-energy parameters are computed. We find that the new contributions demonstrate the arbitrariness of the heavy-Higgs theory but they fail to restore the uniqueness. The $\beta$ parameter is derived in sect. 4 via the calculation of the longitudinal vector boson scattering amplitude for model $\mathrm{B}$, in the heavy-Higgs limit. We assume that the difference between the masses of the scalars is small, within $10 \%$ of the value of the lower mass. We find a small but positive enhancement in the value of $\beta$. The overall result indicates that, for the chosen range of the parameters, no resonance will appear at any energy. A discussion of the corresponding results is presented at the end of sects. 3 and 4 . All computations were done in terms of the algebraic manipulation program SCHOONSCHIP.

\section{Gauge invariant coupling of a scalar singlet particle}

In this section we look for the most general potential describing the coupling of a new scalar and $S U(2) \times U(1)$ singlet particle, $U$, to the Higgs sector of the standard model. A gauge invariant coupling may quite generally be written as:

$$
V=a K^{\dagger} K+b U^{2}+c\left(K^{\dagger} K\right)^{2}+d U^{4}+e\left(K^{\dagger} K\right) U^{2}+h,
$$

where $a, b, c, d, e, h$ are real parameters and $K$ is the conventional Higgs doublet. Here we implied the discrete symmetry $U \rightarrow-U$ (in accordance with the symmetry $H \rightarrow-H$ of the minimal sector). If we further require that the potential has a ground state, i.e., if

$$
e^{2}-4 c d<0, \quad c>0 \text { and } d>0,
$$

then the potential may be written in the form of a sum of squares:

$$
V=a_{1}^{2}\left(K^{\dagger} K-f_{1}^{2}\right)^{2}+\left[b_{1}\left(K^{\dagger} K-f_{1}^{2}\right)+b_{2}\left(U^{2}-f_{2}^{2}\right)\right]^{2},
$$

where the new parameters are given by

$$
\begin{aligned}
a_{1} & =\frac{\sqrt{4 c d-e^{2}}}{2 \sqrt{d}}, \\
b_{1} & =\frac{e}{2 \sqrt{d}}, b_{2}=\sqrt{d} \\
f_{1}^{2} & =\frac{e b-2 a d}{4 c d-e^{2}}, \quad f_{2}^{2}=\frac{a e-2 c b}{4 c d-e^{2}} \\
h & =c f_{1}^{4}+d f_{2}^{4}+e f_{1}^{2} f_{2}^{2} .
\end{aligned}
$$


To the original Higgs sector, represented by the first term on the right-hand side of eq. (2.3), we added a general gauge-invariant interacting term.

The solutions for the minima of the potential of eq. (2.3) are listed below. We are interested in the cases where the doublet field has a non-zero vacuum expectation value or else, there is no mass generation for the vector bosons and the fermions.

(i) If $f_{2}^{2}<0$ and $f_{1}^{\prime 2} \equiv f_{1}^{2}-b_{1} b_{2} f_{2}^{2} /\left(a_{1}^{2}+b_{1}^{2}\right)>0$, only the Higgs doublet develops a non-zero vacuum expectation value: $U_{0}^{2}=0$ and $K_{0}^{\dagger} K_{0}=f_{1}^{\prime 2}$. This will be referred to as model $\mathrm{A}$. Spontaneous breaking of the symmetry gives rise to the following lagrangian:

$$
\begin{aligned}
\mathscr{L}_{(\text {model A })}= & \mathscr{L}_{\mathrm{SU} 2 \times \mathrm{U} 1}-\frac{1}{2}\left(\partial_{\mu} U\right)^{2}-\frac{1}{2} m_{\mathrm{u}}^{2} U^{2}-g g_{\mathrm{u}} r M U^{2} H \\
& -\frac{1}{4} g^{2} g_{\mathrm{u}} r U^{2}\left(H^{2}+\varphi^{2}\right)-g_{\mathrm{s}}^{2} U^{4},
\end{aligned}
$$

where we have defined

$$
\begin{aligned}
& m^{2}=4\left(a_{1}^{2}+b_{1}^{2}\right) f_{1}^{\prime 2}, \quad m_{\mathrm{u}}^{2}=4 \frac{a_{1}^{2} b_{2}^{2}}{a_{1}^{2}+b_{1}^{2}}\left(-f_{2}^{2}\right) \\
& g_{\mathrm{s}}^{2}=b_{2}^{2}, \quad M=\frac{f_{1}^{\prime} g}{\sqrt{2}} .
\end{aligned}
$$

The term $\mathscr{L}_{\text {SU2 } \times \text { Ul }}$ designates the minimal standard model lagrangian. Here, a particular choice is made for the $\mathrm{U}-\mathrm{H}$ interaction coupling constant. In accordance with the Higgs self-interaction coupling constant, it depends on the Higgs mass:

$$
b_{1} b_{2}=\gamma g^{2}=\frac{1}{4} g_{\mathrm{u}} r g^{2}, \quad \text { where } r=\frac{m^{2}}{4 M^{2}}
$$

This is precisely the model introduced in ref. [7].

(ii) If $f_{2}^{2}>0$ and $f_{1}^{2}>0$, both scalars develop non-zero vacuum expectation values: $U_{0}^{2}=f_{2}^{2}$ and $K_{0}^{\dagger} K_{0}=f_{1}^{2}$. This case will be named model B. After spontaneous symmetry breaking there appears a $\mathrm{U}-\mathrm{H}$ transition. The lagrangian is

$$
\begin{aligned}
\mathscr{L}_{(\text {model B })}= & \mathscr{L}_{\mathrm{SU} 2 \times \mathrm{U} 1}-\frac{1}{2}\left(\partial_{\mu} H\right)^{2}-\frac{1}{2}\left(\partial_{\mu} U\right)^{2}-\frac{1}{2} m_{\mathrm{H}}^{2} H^{2}-\frac{1}{2} m_{\mathrm{u}}^{2} U^{2}-4 g_{i} \alpha M^{2} U H \\
& -\frac{1}{8} \alpha g^{2}\left(H^{2}+\varphi^{2}\right)^{2}-\alpha g M\left(H^{2}+\varphi^{2}\right) H-\frac{1}{8} \beta g_{\mathrm{s}}^{2} g^{2} U^{4}-\beta g_{\mathrm{s}} g M U^{3} \\
& -\frac{1}{4} g_{i} \alpha g_{\mathrm{s}} g^{2}\left(H^{2}+\varphi^{2}\right) U^{2}-g_{i} \alpha g M\left(H^{2}+\varphi^{2}\right) U-g_{i} \alpha g_{\mathrm{s}} g M H U^{2},
\end{aligned}
$$


where

$$
\begin{gathered}
m_{\mathrm{H}}^{2}=4\left(a_{1}^{2}+b_{1}^{2}\right) f_{1}^{2}, \quad m_{\mathrm{u}}^{2}=8 b_{2}^{2} f_{2}^{2}, \\
\alpha=\frac{m_{\mathrm{H}}^{2}}{4 M^{2}}, \quad \beta=\frac{m_{\mathrm{u}}^{2}}{4 M^{2}}, g_{\mathrm{s}}=\sqrt{2} \frac{f_{1}}{f_{2}} .
\end{gathered}
$$

The $\mathrm{W}$ mass is specified by the vacuum expectation value of the $H$ field: $M^{2}=g^{2} f_{1}^{2} / 2$. The coupling of the $\mathrm{U}$ particle to the minimal Higgs system has been chosen in a way that it resembles the self coupling of the $\mathrm{H}$ field:

$$
b_{1} b_{2}=\frac{1}{\sqrt{2}} \gamma g_{\mathrm{s}} g^{2}, \quad \text { with } \gamma=\frac{1}{2 \sqrt{2}} g_{i} \frac{m_{\mathrm{H}}^{2}}{4 M^{2}} .
$$

An important characteristic of this lagrangian is the mixing, of zeroth order in $g$, between the fields $U$ and $H$. As a result, both diagonal states couple to the gauge bosons and to the fermions.

It may be interesting to notice that model $\mathrm{A}$ is not a particular case of model $\mathrm{B}$. They become identical in the limiting case:

(iii) If $f_{2}^{2}=0$ the vacuum expectation values become: $U_{0}^{2}=0$ and $K_{0}^{\dagger} K_{0}=f_{1}^{2}>$ 0 . After spontaneous breaking one of the eigenstates is massless and therefore, this case is of no interest.

To complete the study of the extended potentials, it may be interesting to examine a potential without the symmetry $U \rightarrow-U$ :

$$
V=a K^{\dagger} K+b U^{2}+c\left(K^{\dagger} K\right)^{2}+d U^{4}+e\left(K^{\dagger} K\right) U^{2}+k K^{\dagger} K U+l U^{3}+h .
$$

In this case the minimization conditions among the various parameters become quite complicated. To simplify the situation one may start from a potential in the form of a sum of square terms, for instance:

$$
V=a_{1}^{2}\left(K^{\dagger} K+a_{2}\right)^{2}+\left(b_{1} K^{\dagger} K+b_{2} U^{2}+b_{3} U+b_{4}\right)^{2} .
$$

The consequences of such a model will not be examined here. We would like to mention that the model of eq. (2.8) with $b_{2}=b_{4}=0$ has been studied in the past [17].

\section{U particle effects upon low-energy parameters}

The models presented previously will be examined in this section with respect to low-energy parameters, measured at energies below $100 \mathrm{GeV}$. The computations will be done in the large-Higgs-mass limit, with emphasis given to the effects of the 
models in the limit where the $U$ particle becomes as heavy as the Higgs. In connection to the arbitrariness of the large-Higgs-mass limit, an attempt will be made to restore the correspondence between the linear and non-linear $\sigma$-models via the U-particle effects. As mentioned in the introduction, while both models give an equivalent description of the heavy-Higgs theory at the tree- and one-loop levels, this is not the case at the two-loop level. It has been pointed out that the ambiguity in the two-loop results can be attributed, at least partly, to the absence of Higgs self interactions in the non-linear $\sigma$-model [5]. The questionable (quadratic in $m$ ) terms of the linear $\sigma$-model seem to have a specific signature, in the sense that they contain the transcedental numbers $\pi$ or a Clausen function $\mathrm{Cl}(\pi / 3)$ and they can be traced back to the diagrams where they originated. By choosing the interaction of the $U$ field with the Higgs to be similar in form with the Higgs self interactions (eqs. (2.5)-(2.7)), we may be able to generate new corrections of the same form and thus, obtain a cancellation that will simplify or completely restore the correspondence with the nonlinear $\sigma$-model at the two-loop level. Of course if this program were to succeed, such an investigation should be carried on to higher order corrections. It may be interesting to notice that if we take the strong-coupling limit $\left(a_{1}^{2} \rightarrow \infty\right)$ of the extended potential given in eq. (2.3) at the lagrangian level, the $U$ particle decouples.

\subsection{ONE-LOOP RENORMALIZATION FOR MODEL A}

We study model A described by the lagrangian of eq. (2.4). We follow the renormalization scheme explained in detail in ref. [5]. It involves two steps. The first step includes the calculation of the following:

(i) All infinities (poles in $n$-4) of all two-point and three-point functions.

(ii) The complete set of tadpole diagrams.

(iii) All terms behaving like $\mathrm{m}^{2}$ for the two-point and three-point functions.

The next step consists in absorbing all the above terms into the rescalings of fields and parameters, given by

$$
\begin{aligned}
W_{\mu}^{+} & \rightarrow W_{\mu}^{+}\left(1+\delta_{c}\right), \\
A_{\mu} & \rightarrow A_{\mu}\left(1+\delta_{A}\right)+\delta_{A 0} W_{\mu}^{0}, \quad W_{\mu}^{0} \rightarrow W_{\mu}^{0}\left(1+\delta_{0}\right)+\delta_{0 A} A_{\mu}, \\
H & \rightarrow H\left(1+\delta_{H}\right)+\frac{M}{g} \delta_{t}, \quad \varphi \rightarrow \varphi\left(1+\delta_{H}\right), \\
M & \rightarrow M\left(1+\delta_{M}\right), \quad m \rightarrow m\left(1+\delta_{m}\right), \\
c & \rightarrow c\left(1+\delta_{c}\right), \quad g \rightarrow g\left(1+\delta_{g}\right)
\end{aligned}
$$


In order to account for the contributions of the $U$ particle, the $\delta$ 's, found in the standard model [5], have to be modified in the following way:

$$
\begin{aligned}
\delta_{t} \rightarrow & \delta_{t}\left(\mathrm{SU}_{2} \times \mathrm{U}_{1}\right)+\frac{g^{2}}{8 \pi^{2}(n-4)}\left(\frac{1}{4} \frac{m^{2}}{M^{2}} g_{\mathrm{u}}+\frac{1}{4} g_{\mathrm{u}}^{\prime}\right) \\
& +\frac{g^{2}}{8 \pi^{2}}\left(-\frac{1}{8} \frac{m^{2}}{M^{2}} g_{\mathrm{u}}+\frac{1}{8} \frac{m^{2}}{M^{2}} g_{\mathrm{u}} \ln \left(m^{2}\right)-\frac{1}{8} g_{\mathrm{u}}^{\prime}+\frac{1}{8} g_{\mathrm{u}}^{\prime} \ln \left(M^{2}\right)\right) \\
& +\frac{g^{2}}{8 \pi^{2}}(n-4)\left(\frac{1}{16} \frac{m^{2}}{M^{2}} g_{\mathrm{u}}-\frac{\pi^{2}}{192} \frac{m^{2}}{M^{2}} g_{\mathrm{u}}-\frac{1}{16} \frac{m^{2}}{m^{2}} g_{\mathrm{u}} \ln \left(m^{2}\right)\right. \\
& \left.+\frac{1}{32} \frac{m^{2}}{M^{2}} g_{\mathrm{u}} \ln { }^{2}\left(m^{2}\right)+\frac{1}{16} g_{\mathrm{u}}^{\prime}+\frac{\pi^{2}}{192} g_{\mathrm{u}}^{\prime}-\frac{1}{16} g_{\mathrm{u}}^{\prime} \ln \left(M^{2}\right)+\frac{1}{32} g_{\mathrm{u}}^{\prime} \ln ^{2}\left(M^{2}\right)\right) \\
\delta_{2} \rightarrow & \delta_{2}\left(\mathrm{SU}_{2} \times \mathrm{U}_{1}\right)+\frac{g^{2}}{8 \pi^{2}(n-4)}\left(-\frac{1}{16} \frac{m^{2}}{M^{2}} g_{\mathrm{u}}^{2}-\frac{1}{16} \frac{m^{2}}{M^{2}} g_{\mathrm{u}}^{\prime 2}\right) \\
& \left.+\frac{1}{82} \frac{m^{2}}{M^{2}} g_{\mathrm{u}}^{\prime 2} \ln \left(m^{2}\right)+\frac{1}{16} \frac{m^{2}}{M^{2}} g_{\mathrm{u}}^{\prime 2}\right] \\
& \frac{1}{32} \frac{m^{2}}{M^{2}} g_{\mathrm{u}}^{2}\left(\frac{\pi}{\sqrt{3}}-2\right)-\frac{1}{32} \frac{m^{2}}{M^{2}} g_{\mathrm{u}}^{2} \ln \left(m^{2}\right)
\end{aligned}
$$

while the rest of the standard model shifts remain unaltered. Substitution of the rescaled quantities in the gauge invariant lagrangian reproduces all infinities and $m^{2}$ terms at one-loop level. The terms with $g_{u}$ and $g_{u}^{\prime}$ refer to heavy and light U, respectively. Also, $\delta_{2} \equiv \delta_{M}-\delta_{m}$.

For the specification of $\delta_{t}$ we required the subtraction of the whole tadpole, including the terms proportional to $\epsilon=n-4$. Omission of this latter step would introduce false $m^{2} g_{\mathrm{u}}$ contributions to the leptonic process at two-loop. Also, $\delta_{2}$ is determined in a way that the lagrangian parameter $m$ is the physical mass of the Higgs particle (location of pole of propagator). As a consequence of this, the only $\mathrm{m}^{2}$ terms remaining, after the one-loop subtraction, are to be found in the Higgs self energy. The renormalization of the new coupling constants $g_{u}$ and $g_{s}$ is not presented here, since it does not enter the present calculations. 
3.2. MODEL A. RADIATIVE CORRECTIONS TO THE $\rho$ PARAMETER AND THE VECTOR BOSONS MASS SHIFTS

(i) The $\rho$ parameter. It is defined as the ratio of the strengths of a neutral and a charged current process, at low energy. As such, it is customary to consider $\nu_{\mu} \mathrm{e}$ scattering and $\mu$ decay, respectively, at zero energy and momentum transfer.

We will calculate the leading, in the Higgs mass, radiative corrections to the $\rho$ parameter caused by the introduction of the $U$ particle described by the lagrangian of eq. (2.4). Diagrammatically speaking, the U-particle effects appear in the form of self-energy insertions to the Higgs lines. Given that the coupling of the Higgs fields to the leptons is very weak (proportional to $M_{\text {lepton }} / M_{\mathrm{W}}$ ), the relevant diagrams are the ones in which the Higgs interacts only with the vector bosons. Thus the U-dependent corrections arise first at the two-loop level and in particular through irreducible two-loop vector boson self-energy insertions. As far as reducible diagrams with $U$ dependence are concerned, they always involve tadpoles which cancel against diagrams with counterterms, since the whole tadpole is subtracted in the one-loop renormalization. Finally, the diagrams containing twoloop tadpoles will not be computed since they couple to the charged and neutral vector bosons lines with a relative strength 1 and $1 / c^{2}$ respectively and therefore, do not contribute to the $\rho$ parameter.

The irreducible vector boson self-energy diagrams with U-particle effects fall into four different topologies, shown in fig. 1. From these, due to the subtraction of the $m^{2}$ terms at the one-loop level, the disjoint diagrams of figs. $1 \mathrm{c}, 1 \mathrm{~d}$ cancel against the linear-in- $g_{\mathrm{u}}$ terms of the one-loop diagrams with counterterms. Consequently, no $\mathrm{U}$-dependent corrections on the photon-photon and photon- $\mathrm{W}_{0}$ amplitudes are obtained. All new corrections to the vector boson self energies are shown in fig. 2. The crosses stand for one-loop counterterms with $g_{\mathrm{u}}^{2}$ dependence. It should be noted that additional counterterms proportional to $g^{4}$ arise when we perform the shifts mentioned earlier in two parameters or fields of the same term of the lagrangian. These must be kept at the two-loop level and they contribute to the self energies. However, the U-dependent such contributions do not contribute
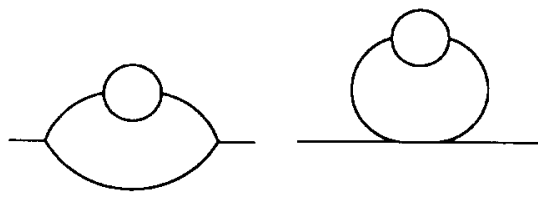

$-\mathbf{a}-$

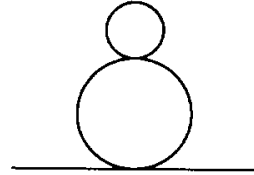

$-c-$

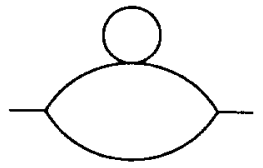

$-d-$

Fig. 1. Topologies for two-loop irreducible vector boson self-energy diagrams with U dependence. 


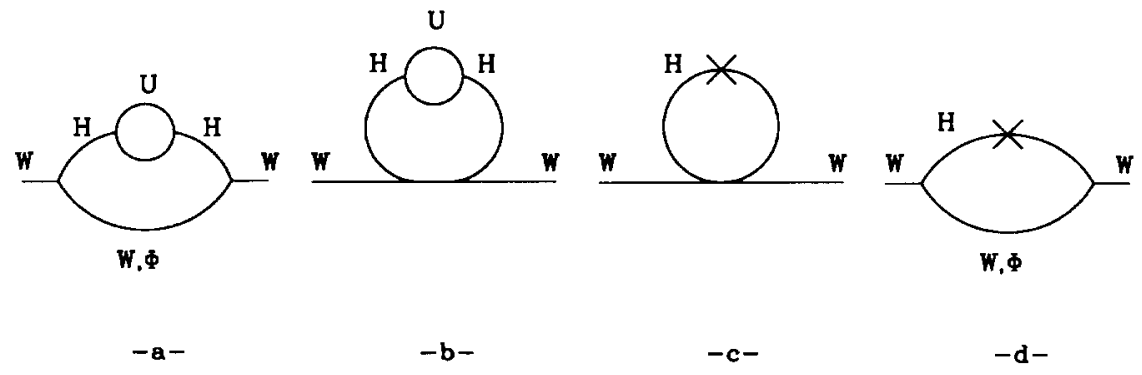

Fig. 2. Diagrams contributing to the $\rho$ parameter and the $W$ mass shifts.

to the $\rho$. Details of the calculations are given in appendix A. The corrections to the $\rho$ parameter are given by

$$
\delta \rho=\frac{1}{(2 \pi)^{4} i} \frac{c^{2} \Pi^{0}\left(p^{2}=0\right)}{M^{2}}-\frac{1}{(2 \pi)^{4} i} \frac{\Pi^{c}\left(p^{2}=0\right)}{M^{2}},
$$

where $\Pi^{\mathrm{c}, 0}$ denote the charged and neutral vector boson self energies. The result for light $\mathrm{U}$ is

$$
\delta \rho=0+\mathrm{O}\left(\ln \left(m^{2}\right)\right), \quad \text { if } m_{\mathrm{u}}=M
$$

and thus, no information on light $\mathrm{U}$ matter can be derived through measurements of $\rho$. For heavy $\mathrm{U}$, the leading corrections grow like $m^{2}$, as follows:

$$
\delta \rho=g^{4} g_{\mathrm{u}}^{2} \frac{m^{2}}{M^{2}} \tan ^{2} \theta \frac{1}{8192 \pi^{4}}\left[-\frac{12}{\sqrt{3}} \mathrm{Cl}\left(\frac{\pi}{3}\right)+\frac{3 \pi}{\sqrt{3}}\right], \quad \text { if } m_{\mathrm{u}}=m .
$$

At this point, the fitting of the free parameters $M, s_{\theta}$ and $g$ to the experimental data must be performed by means to low-energy leptonic processes, for instance, $\mu$ decay (to define the Fermi constant), a neutral current process i.e., $\nu_{\mu}$ e scattering (to defined the weak mixing angle) and Coulomb scattering of muons on electrons (to define $\alpha=e^{2} / 4 \pi$ ) [18]. For the present calculation, the tree-level fitting is appropriate, which gives the experimental or tree-level quantities, as follows:

$$
\begin{gathered}
G_{\mathrm{F}}=\frac{g_{\exp }^{2} \sqrt{2}}{8 M_{\exp }^{2}}, \quad \alpha=\frac{e^{2}}{4 \pi}=\frac{g_{\exp }^{2} s_{\exp }^{2}}{4 \pi}, \\
\frac{\sigma^{\bar{\nu} \mathrm{e}}}{\sigma^{\nu \mathrm{e}}}=\frac{\xi^{2}-\xi+1}{\xi^{2}+\xi+1}, \quad \text { with } \xi=1-4 s_{\exp }^{2} .
\end{gathered}
$$

Hence, eq. (3.2), with the experimental low-energy values substituted for the parameters, gives all the effects of a heavy $U$ particle. The result will be discussed in section 3.4 . 
(ii) The $W$ mass shifts. The dominant, quadratic in $m$, contributions of the new scalar $U$ to the mass shift of the vector bosons will now be considered. The mass shift is defined as the difference between the values of the "on-shell" mass (position of the pole of the propagator) and the "experimental" mass (defined in (3.3)):

$$
\delta M=\frac{\bar{M}^{2}-M_{\mathrm{exp}}^{2}}{2 M-} \quad \text { and } \quad \delta M_{0}=\frac{\bar{M}_{0}^{2}-M_{0, \exp }^{2}}{2 M_{0}}
$$

where $p^{2}=-\bar{M}^{2}$ and $p^{2}=-\bar{M}_{0}^{2}$ are the positions of the pole of the charged and neutral $\mathrm{W}$ propagators, respectively. The new contributions with $\mathrm{U}$ dependence are given by

$$
\begin{aligned}
& \bar{M}^{2}-M_{\text {exp }}^{2}=-\left[A_{c}\left(p^{2}=-M^{2}\right)-A_{c}\left(p^{2}=0\right)\right], \\
& \bar{M}_{0}^{2}-M_{0, \exp }^{2}=-\left[A_{0}\left(p^{2}=-M_{0}^{2}\right)-A_{0}\left(p^{2}=0\right)+M_{0, \exp }^{2} \delta \rho\right],
\end{aligned}
$$

where we have written the irreducible vector boson self-energy diagrams as

$$
\Pi_{\mu \nu}^{\mathrm{c}, 0}\left(p^{2}\right)=(2 \pi)^{4} i\left[A_{\mathrm{c}, 0}\left(p^{2}\right) \delta_{\mu \nu}+B_{\mathrm{c}, 0}\left(p^{2}\right) p_{\mu} p_{\nu}\right]
$$

The subscripts $\mathrm{c}$ and 0 refer to charged and neutral bosons, respectively. The U-particle effects appear at the two-loop level in diagrams shown in figs. $2 \mathrm{a}, 2 \mathrm{c}$. Details of the calculation are given in appendix $\mathrm{A}$.

In the case of a light $\mathrm{U}$ particle, $m_{\mathrm{u}}=M$, the quadratic contributions to the mass shifts are zero. The leading dependence is logarithmic in $m$. For heavy U, $m_{\mathrm{u}}=m$, we find

$$
\begin{aligned}
\delta M & =-g^{4} g_{\mathrm{u}}^{2} \frac{m^{2}}{M^{2}} \frac{1}{16384 \pi^{4}}\left[-\frac{4}{3 \sqrt{3}} \mathrm{Cl}\left(\frac{\pi}{3}\right)+\frac{\pi}{3 \sqrt{3}}\right], \\
\delta M_{0} & =-g^{4} g_{\mathrm{u}}^{2} \frac{m^{2}}{c^{3} M} \frac{1}{16384 \pi^{4}}\left[-\frac{4}{3 \sqrt{3}} \mathrm{Cl}\left(\frac{\pi}{3}\right)+\frac{\pi}{3 \sqrt{3}}\right]-\frac{1}{2} \frac{M}{c} \delta \rho \\
& =-g^{4} g_{\mathrm{u}}^{2} \frac{m^{2}}{c^{3} M}\left(9 s^{2}+1\right) \frac{1}{16384 \pi^{4}}\left[-\frac{4}{3 \sqrt{3}} \mathrm{Cl}\left(\frac{\pi}{3}\right)+\frac{\pi}{3 \sqrt{3}}\right],
\end{aligned}
$$

where the free parameters are to be replaced by their experimental values. See sect. 3.4 for a discussion of the result. 


\subsection{THE $\rho$ PARAMETER FOR MODEL B}

We examine the consequences of model $\mathrm{B}$ described by the lagrangian of eq. (2.6), as far as the cancellation of the ambiguous terms is concerned. For this, we compute the new corrections to the $\rho$ parameter.

The calculation is done in terms of the non-diagonal fields $H$ and $U$. At the one-loop level, there are no vector boson self-energy diagrams involving the $U$ field. However, the presence of the new scalar is manifested through the change of the propagator for the $\mathrm{H}$ field (see appendix $\mathrm{B}$ ). The one-loop result is found:

$$
\delta \rho=-\frac{3 g^{2}}{64 \pi^{2}} \tan ^{2} \theta\left[\ln \left(\frac{m_{-}^{2}}{M^{2}}\right)+y \ln \left(\frac{m_{+}^{2}}{m_{-}^{2}}\right)\right]
$$

where $\theta$ is the weak mixing angle and $y$ is defined in appendix $B$. The first term on the right-hand side is precisely the standard model result with the original Higgs mass substituted by the lower mass eigenvalue $m_{-}$. The second term in eq. (3.7) represents the new effects arising from the extension of the scalar sector. Notice that this term may become arbitrarily large as the ratio $m_{+}^{2} / m_{-}^{2}$ grows. Thus, the reported correspondence: $\ln \left(m^{2}\right)$ [linear model] $\rightarrow 2 /(n-4)$ [non-linear model] is violated even at the one-loop level. It may be worth noticing here that at the two-loop level the complications become even worse. New types of Clausen functions with arguments different than $\pi / 3$ enter the calculations. In this sense the ambiguity is increased.

\subsection{DISCUSSION OF THE LOW-ENERGY RESULTS}

We have computed the two-loop effects of the U particle of model A at low energies. The result indicates that light $U$ matter gives weak (at most logarithmic in $m$ ), corrections to the $\rho$ and the vector boson shifts. In the case of a heavy $\mathrm{U}$ particle, the radiative corrections to the $\rho$ parameter and to the $\mathrm{W}$ mass shifts have a leading dependence which is quadratic in the Higgs mass at the two-loop level. While the new effects are very small for direct observation, their occurrence demonstrates that the $\mathrm{U}$ particle is a suitable means of parametrizing the ambiguous terms in the heavy-Higgs limit, as suggested in ref. [7]. It also indicates that the decoupling theorem [19] does not apply in the case of heavy scalars interacting with a strength proportional to the square of the heavy mass. However, the restoration of the correspondence between linear and non-linear $\sigma$-models is not possible and model A must be dismissed in this respect. In fact, concerning the $\rho$ parameter, if we compare our result of eq. (3.2) with the corresponding one for the standard model [5] we observe a striking similarity in form. Apart from an overall factor 9 (a combinatorial factor), our result reproduces the terms containing the Clausen function and the $\pi / \sqrt{3}$ which, as mentioned earlier, are precisely the 


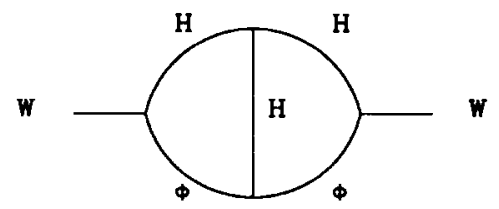

Fig. 3. Additional diagram contributing $\mathrm{Cl}(\pi / 3)$ terms in the standard model.

ambiguous terms. Unfortunately, these terms appear with the same sign in both calculations and, since they are quadratic in $g_{u}$, the cancellation cannot occur. In the results for the mass shifts, even this analogy in form is lost. While terms with the Clausen function appear in eqs. (3.5) and (3.6), no such effects arise in the standard model result [6] due to the existence of an extra source of Clausen functions in the diagram of fig. 3. No corresponding topology exists in the enlarged model. Consequently, the elimination of the ambiguous $m^{2}$ terms with this model is not merely a sign problem but it also involves a discrepancy in structure. However, even if we had succeeded in exactly duplicating the ambiguous terms in all observable quantities in terms of another model, the problem would not have been solved. This study revealed that the greatest difficulty stems from the double occurrence of the new coupling constant $g_{u}$. This seems inherent in all models. For this reason, we tried to create a model with a fermionic U-particle. The idea was that a fermionic loop would provide the extra minus sign needed. In fact it is possible to construct a sensible model with an ultra-heavy fermionic singlet coupled to the Higgs sector (by means of the see-saw mechanism) but unfortunately it does not produce the appropriate quadratic effects in the large mass.

The study of model $B$ in sect. 3 indicated that models with a mixing between the scalars further complicate the relation between the linear and non-linear $\sigma$-models. The extended Higgs sector manifested itself even at the one-loop level with corrections that may become significant if the difference between the two mass scales is large.

The above analysis covers all extensions of the minimal theory via the addition of a singlet with the symmetry $U \rightarrow-U$. Moreover, it certainly discourages further investigation of models without this discrete symmetry. Thus, the results of a heavy and strongly interacting scalar sector remain arbitrary.

\section{4. $U$ particle effects upon $W_{L} W_{L}$ scattering and the $\beta$ parameter}

We now focus on the effects of the extended scalar sector at energies higher than the mass of the W's and even higher scalar masses. At first we compute the radiative corrections to the $\mathrm{W}_{\mathrm{L}} \mathrm{W}_{\mathrm{L}} \rightarrow \mathrm{W}_{\mathrm{L}} \mathrm{W}_{\mathrm{L}}$ scattering amplitude which is expected to play a decisive role in revealing the nature of the electroweak symmetry breaking sector. In case the Higgs is heavy, it has been suggested that the 
prominent signal for the new multi-TeV hadron colliders will come from the WW fusion [3]. Next, following the treatment of ref. [14], we derive the Lehmann $\beta$ parameter from the one-loop $\mathrm{W}_{\mathrm{L}} \mathrm{W}_{\mathrm{L}}$ scattering amplitude calculated below the threshold. While the dynamics of the strongly interacting sector are unknown, this approach extrapolates the lower energy results, in accordance with partial wave analysis methods developed in the study of $\pi \pi$ scattering [15]. The $\beta$ parameter designates the position of dynamical resonances in the $\mathrm{TeV}$ regime where the perturbative treatment is not valid. When computed in the heavy-Higgs limit, $\beta$ is sensitive to the way the limit is taken, a fact which may be demonstrated by its dependence on the $U$ particle of model A [14]. Here we will examine the $U$ particle effects upon the position of resonances for the particular case of model $\mathrm{B}$. We will work in the limit where $m_{ \pm}$are infinitely large but with values close together, i.e., without introducing a mass hierarchy in the heavy sector.

\subsection{THE MODEL}

A simplified version of model B (see eq. (2.6)) will be used to compute the radiative corrections to the longitudinal WW scattering amplitude at the one-loop level. We consider an SU(2) gauge theory which is the limit of the standard model for zero weak mixing angle. The gauge invariant lagrangian is given by

$$
\begin{aligned}
\mathscr{L}_{\mathrm{GI}}= & -\frac{1}{4}\left(\partial_{\mu} W_{\nu}^{a}-\partial_{\nu} W_{\mu}^{a}+g \epsilon_{a b c} W_{\mu}^{b} W_{\nu}^{c}\right)^{2}-\frac{1}{2} M^{2} W^{2}-\frac{1}{2}\left(\partial_{\mu} \phi^{a}\right)\left(\partial_{\mu} \phi^{a}+g \epsilon_{a b c} W_{\mu}^{b} \phi^{c}\right) \\
& +\frac{1}{2} g W_{\mu}^{a}\left(H \partial_{\mu} \phi^{a}-\phi^{a} \partial_{\mu} H\right)-\frac{1}{2} g M W^{2} H-\frac{1}{8} g^{2} W_{\mu}^{2}\left(H^{2}+\phi^{2}\right)-M \phi^{a} \partial_{\mu} W_{\mu}^{a} \\
& -\frac{1}{2}\left(\partial_{\mu} H\right)^{2}-\frac{1}{2}\left(\partial_{\mu} U\right)^{2}-\frac{1}{2} m_{\mathrm{H}}^{2} H^{2}-\frac{1}{2} m_{\mathrm{u}}^{2} U^{2}-4 g_{i} \alpha M^{2} U H \\
& -\frac{1}{8} \alpha g^{2}\left(H^{2}+\phi^{2}\right)^{2}-\alpha g M\left(H^{2}+\phi^{2}\right) H-\frac{1}{8} \beta g_{\mathrm{s}}^{2} g^{2} U^{4}-\beta g_{\mathrm{s}} g M U^{3} \\
& -\frac{1}{4} g_{i} \alpha g_{\mathrm{s}} g^{2}\left(H^{2}+\phi^{2}\right) U^{2}-g_{i} \alpha g M\left(H^{2}+\phi^{2}\right) U-g_{i} \alpha g_{\mathrm{s}} g M H U^{2},
\end{aligned}
$$

where $\alpha=m_{\mathrm{H}}^{2} / 4 M^{2}$ and $\beta=m_{\mathrm{u}}^{2} / 4 M^{2}$. The complete lagrangian includes a gauge fixing term and a ghost lagrangian. The Feynman-'t Hooft gauge is chosen. The Feynman rules are given in appendix $\mathrm{B}$.

For reasons that will be explained in sect. 4.3 the existence of two different mass scales in the scalar sector introduces considerable technical difficulties in the computation at hand. For this reason we make the following assumptions which simplify the calculations while still illustrating the general properties of the model. In general we may have $m_{\mathrm{u}}^{2} \propto m_{\mathrm{H}}^{2}$. The simplest choice is

$$
m_{\mathrm{H}}^{2}=m_{\mathrm{u}}^{2}=m^{2} \text {. }
$$


As a consequence, the scalar mass eigenvalues and eigenstates become at the tree level:

$$
m_{ \pm}^{2}=m^{2}\left(1 \pm\left|g_{i}\right|\right) \text { and } \Omega_{ \pm}=\frac{1}{\sqrt{2}}\left(H \pm \frac{\left|g_{i}\right|}{g_{i}} U\right),
$$

where $\left|g_{i}\right|<1$. According to eq. (4.3), the coupling $g_{i}$ is a measure of the difference between the two large scalar masses. We make the additional assumption that this difference is small:

$$
\left|g_{i}\right| \ll 1,
$$

and calculate the corrections to the WW scattering that are linear in $g_{i}$. Notice that the size of $g_{i}$ does not affect the strength of the U-H coupling since the latter also depends on $g_{\mathrm{s}}$. Thus the model describes two heavy scalars of approximately the same size interacting strongly. As free parameters in the scalar sector we take $m_{-}$and $g_{i}$, while an expansion in $g_{i}$ yields

$$
\begin{aligned}
& m_{+}^{2}=m_{-}^{2}\left(1+2\left|g_{i}\right|+\mathrm{O}\left(g_{i}^{2}\right)\right), \\
& \Delta_{\mathrm{H}}=\frac{1}{2}\left(\frac{1}{k^{2}+m_{+}^{2}}+\frac{1}{k^{2}+m_{-}^{2}}\right)=\frac{1}{k^{2}+m_{-}^{2}}\left(1-\left|g_{i}\right| \frac{m_{-}^{2}}{k^{2}+m_{-}^{2}}+\mathrm{O}\left(g_{i}^{2}\right)\right) .
\end{aligned}
$$

In this sense the parameter $m_{\mathrm{H}}$, which plays the role of the cutoff in the standard model, is now substituted by $m_{-}$and the presence of extra scalar is indicated by the linear-in- $g_{i}$ effects.

\subsection{ONE-LOOP RENORMALIZATION}

The renormalization scheme may be outlined as follows: As a first step one must rescale the fields and parameters in the gauge invariant lagrangian:

$$
\begin{gathered}
W_{\mu}^{a} \rightarrow W_{\mu}^{a}\left(1+\delta_{w}\right), \quad M \rightarrow M\left(1+\delta_{M}\right), \\
g \rightarrow g\left(1+\delta_{g}\right), \quad \phi \rightarrow \phi\left(1+\delta_{H}\right), \\
H \rightarrow H\left(1+\delta_{H}\right)+\delta_{H U} U+\frac{M}{g} \delta t, \\
U \rightarrow U\left(1+\delta_{U}\right)+\delta_{U H} H+\frac{M}{g} \delta_{t u}, \\
g_{i} \rightarrow g_{i}\left(1+\delta_{g_{i}}\right), \quad g_{\mathrm{s}} \rightarrow g_{\mathrm{s}}\left(1+\delta_{g_{\mathrm{s}}}\right), \\
m_{H} \rightarrow m_{H}(1+\delta m), \quad \mu \rightarrow \mu(1+\delta \mu),
\end{gathered}
$$

so that $m_{u} \rightarrow m_{u}\left(1+\delta m+\frac{1}{2} \delta \mu\right)$, 
In the above we have introduced the parameter $\mu$ as follows: $m_{\mathrm{H}}^{2} \equiv m^{2}$ and $m_{\mathrm{u}}^{2} \equiv \mu m^{2}$. As mentioned earlier (see eq. (4.2)) we assume at the tree level: $m_{\mathrm{u}, \text { ren }}=m_{\mathrm{H}, \mathrm{ren}}$ or, $\mu_{\mathrm{ren}}=1$. In general this relation is not valid at the one-loop level, hence the necessity to introduce $\delta_{\mu}$. Instead of $\delta_{M}$ we will use: $\delta_{1} \equiv \delta_{M}+\delta_{t} / 2$.

The second step in the renormalization program involves the specification of the various $\delta$ 's in a way that they reproduce the infinities and all the terms proportional to the square of the heavy scalar masses at the one-loop level. Thus the renormalization program requires the calculation of:

(i) the complete set of the tadpole diagrams,

(ii) all infinities and terms quadratic in the heavy masses $m_{ \pm}$of all the two-point functions,

(iii) the infinities of the three-point functions.

As far as the subtraction of the finite (quadratic in the heavy mass) terms is concerned, it is sufficient to compute them only in the self-energy diagrams. It then follows from the Ward identities that the three-point and the four-point diagrams are also free of quadratic dependence in the heavy masses. The argument [5] does not hold for diagrams with $\mathrm{H}$ or $\mathrm{U}$ external lines. The shifts $\delta_{t}$ and $\delta_{t u}$ are specified by the subtraction of the complete tadpole for $\mathrm{H}$ and $\mathrm{U}$.

The terms (i)-(iii) are reproduced up to terms of order $g_{i}^{2}$ by the following values:

$$
\begin{aligned}
\delta_{t}= & \frac{g^{2}}{16 \pi^{2}(n-4)}\left(\frac{3}{2}+9 \frac{M^{2}}{m_{-}^{2}}+\frac{3}{2} \frac{m_{-}^{2}}{M^{2}}-g_{i} g_{\mathrm{s}} \frac{m_{-}^{2}}{M^{2}}-9\left|g_{i}\right| \frac{M^{2}}{m_{-}^{2}}+\frac{3}{2}\left|g_{i}\right| \frac{m_{-}^{2}}{M^{2}}\right) \\
& +\delta_{t \mathrm{f}}, \\
\delta_{t u}= & \frac{g^{2}}{8 \pi^{2}(n-4)}\left(\frac{3}{4} g_{\mathrm{s}} \frac{m_{-}^{2}}{M^{2}}-\frac{9}{2} g_{i} \frac{M^{2}}{m_{-}^{2}}-\frac{1}{2} g_{i} \frac{m_{-}^{2}}{M^{2}}+\frac{3}{4}\left|g_{i}\right| g_{\mathrm{s}} \frac{m_{-}^{2}}{M^{2}}\right)+\delta_{t u, \mathrm{f}}, \\
\delta_{1}= & -\frac{g_{-}^{2}}{16 \pi^{2}(n-4)} \frac{25}{6}+\delta_{1 \mathrm{f}}, \\
\delta_{m}= & \frac{g^{2}}{8 \pi^{2}(n-4)}\left(-\frac{9}{8}+\frac{3}{8} \frac{m_{-}^{2}}{M^{2}}+\frac{1}{4} g_{i} g_{\mathrm{s}} \frac{m_{-}^{2}}{M^{2}}+\frac{3}{8}\left|g_{i}\right| \frac{m_{-}^{2}}{M^{2}}\right)+\delta_{m \mathrm{f}}, \\
\delta_{\mu}= & \frac{g^{2}}{8 \pi^{2}(n-4)}\left(\frac{9}{4}+\frac{3}{8} g_{\mathrm{s}}^{2} \frac{m_{-}^{2}}{M^{2}}-\frac{3}{4} \frac{m_{-}^{2}}{M^{2}}+\frac{9}{2} g_{i} g_{\mathrm{s}} \frac{M^{2}}{m_{-}^{2}}+\frac{3}{8}\left|g_{i}\right| g_{\mathrm{s}}^{2} \frac{m_{-}^{2}}{M^{2}}\right. \\
& \left.-\frac{3}{4}\left|g_{i}\right| \frac{m_{-}^{2}}{M^{2}}\right)+\delta_{\mu \mathrm{f}}
\end{aligned}
$$




$$
\begin{aligned}
\delta_{g_{i}} & =\frac{g^{2}}{8 \pi^{2}(n-4)}\left(\frac{9}{8}-\frac{9}{4} \frac{M^{2}}{m_{-}^{2}}-\frac{3}{8} \frac{m_{-}^{2}}{M^{2}}\right)+\delta_{g_{i}, \mathrm{f}}, \\
\delta_{U H} & =-\delta_{H U}=0 \\
\delta_{U} & =0+\delta_{U \mathrm{f}}, \quad \delta_{H}=\frac{g^{2}}{8 \pi^{2}(n-4)} \frac{3}{4}+\delta_{H \mathrm{f}}, \\
\delta_{w} & =\frac{g^{2}}{8 \pi^{2}(n-4)} \frac{19}{12}, \quad \delta_{g}=-\frac{g^{2}}{8 \pi^{2}(n-4)} \frac{43}{12} .
\end{aligned}
$$

The finite $\delta$ 's (indicated by the index $\mathrm{f}$ ) are chosen in a way that the reproduce the radiative corrections that have a quadratic dependence in the heavy masses $m_{ \pm}$. Their values, up to terms of order $g_{i}^{2}$, are the following:

$$
\begin{aligned}
& \delta_{t \mathrm{f}}=\frac{g^{2}}{16 \pi^{2}(n-4)}\left(-\frac{3}{4}-\frac{3}{2} \frac{M^{2}}{m_{-}^{2}}+\frac{9}{2} \frac{M^{2}}{m_{-}^{2}} \ln \left(M^{2}\right)-\frac{3}{4} \frac{m_{-}^{2}}{M^{2}}+\frac{3}{4} \frac{m_{-}^{2}}{M^{2}} \ln \left(m_{-}^{2}\right)\right. \\
& +\frac{3}{4} \ln \left(M^{2}\right)+\frac{1}{2} g_{i} g_{\mathrm{s}} \frac{m_{-}^{2}}{M^{2}}+\frac{3}{2}\left|g_{i}\right| \frac{M^{2}}{m_{-}^{2}}-\frac{9}{2}\left|g_{i}\right| \frac{M^{2}}{m_{-}^{2}} \ln \left(M^{2}\right) \\
& \left.-\frac{1}{2} g_{i} g_{\mathrm{s}} \frac{m_{-}^{2}}{M^{2}} \ln \left(m_{-}^{2}\right)+\frac{3}{4}\left|g_{i}\right| \frac{m_{-}^{2}}{M^{2}} \ln \left(m_{-}^{2}\right)\right) \\
& \delta_{t u, \mathrm{f}}=\frac{g_{-.}^{2}}{8 \pi^{2}(n-4)}\left(-\frac{3}{8} g_{\mathrm{s}} \frac{m_{-}^{2}}{M^{2}}+\frac{3}{4} g_{i} \frac{M^{2}}{m_{-}^{2}}+\frac{1}{4} g_{i} \frac{m_{-}^{2}}{M^{2}}-\frac{9}{4} g_{i} \frac{M^{2}}{m_{-}^{2}} \ln \left(M^{2}\right)\right. \\
& \left.-\frac{1}{4} g_{i} \frac{m_{-}^{2}}{M^{2}} \ln \left(m_{-}^{2}\right)+\frac{3}{8}\left|g_{i}\right| g_{\mathrm{s}} \frac{m_{-}^{2}}{M^{2}} \ln \left(m_{-}^{2}\right)+\frac{3}{8} g_{\mathrm{s}} \frac{m_{-}^{2}}{M^{2}} \ln \left(m_{-}^{2}\right)\right) \\
& \delta_{m \mathrm{f}}=\frac{g_{-}^{2}}{8 \pi^{2}(n-4)}\left(\frac{3}{16}-\frac{9}{16} \frac{m_{-}^{2}}{M^{2}}+\frac{9}{32} \frac{m_{-}^{2}}{M^{2}} \frac{\pi}{\sqrt{3}}-\frac{1}{8} g_{i} g_{\mathrm{s}} \frac{m_{-}^{2}}{M^{2}}-\frac{3}{8}\left|g_{i}\right| \frac{m_{-}^{2}}{M^{2}}\right. \\
& +\frac{9}{32}\left|g_{i}\right| \frac{m_{-}^{2}}{M^{2}} \frac{\pi}{\sqrt{3}}-\frac{3}{16} \ln \left(M^{2}\right)+\frac{3}{16} \frac{m_{-}^{2}}{M^{2}} \ln \left(m_{-}^{2}\right) \\
& \left.+\frac{1}{8} g_{i} g_{\mathrm{s}} \frac{m_{-}^{2}}{M^{2}} \ln \left(m_{-}^{2}\right)+\frac{3}{16}\left|g_{i}\right| \frac{m_{-}^{2}}{M^{2}} \ln \left(m_{-}^{2}\right)\right)
\end{aligned}
$$




$$
\begin{aligned}
\delta_{\mu \mathrm{f}}= & \frac{g^{2}}{8 \pi^{2}(n-4)}\left(-\frac{3}{8}+\frac{9}{8} \frac{m_{-}^{2}}{M^{2}}-\frac{3}{4} g_{\mathrm{s}}^{2} \frac{m_{-}^{2}}{M^{2}}-\frac{9}{16}\left(1-g_{\mathrm{s}}^{2}\right) \frac{m_{-}^{2}}{M^{2}} \frac{\pi}{\sqrt{3}}+\frac{3}{4}\left|g_{i}\right| \frac{m_{-}^{2}}{M^{2}}\right. \\
& -\frac{9}{16}\left|g_{i}\right| g_{\mathrm{s}}^{2} \frac{m_{-}^{2}}{M^{2}}-\frac{9}{16}\left|g_{i}\right|\left(1-g_{\mathrm{s}}^{2}\right) \frac{m_{-}^{2}}{M^{2}} \frac{\pi}{\sqrt{3}}+\frac{3}{8} \ln \left(M^{2}\right)-\frac{3}{8} \frac{m_{-}^{2}}{M^{2}} \ln \left(m_{-}^{2}\right) \\
& \left.+\frac{3}{16} g_{\mathrm{s}}^{2} \frac{m_{-}^{2}}{M^{2}} \ln \left(m_{-}^{2}\right)-\frac{3}{8}\left|g_{i}\right| \frac{m_{-}^{2}}{M^{2}} \ln \left(m_{-}^{2}\right)+\frac{3}{16}\left|g_{i}\right| g_{\mathrm{s}}^{2} \frac{m_{-}^{2}}{M^{2}} \ln \left(m_{-}^{2}\right)\right) \\
\delta_{g_{i}, \mathrm{f}}= & \frac{g^{2}}{8 \pi^{2}(n-4)}\left(-\frac{3}{16}+\frac{3}{32} g_{\mathrm{s}}^{2} \frac{m_{-}^{2}}{M^{2}}+\frac{15}{16} \frac{m_{-}^{2}}{M^{2}}-\frac{9}{16} \frac{m_{-}^{2}}{M^{2}} \frac{\pi}{\sqrt{3}}\right. \\
& \left.+\frac{3}{16} \ln \left(M^{2}\right)-\frac{3}{16} \frac{m_{-}^{2}}{M^{2}} \ln \left(m_{-}^{2}\right)\right), \\
\delta_{U \mathrm{f}}= & \frac{g^{2}}{8 \pi^{2}(n-4)}\left(1+\left|g_{i}\right|\right)\left(\frac{13}{32} \frac{m_{-}^{2}}{M^{2}}-\frac{9}{32} g_{\mathrm{s}}^{2} \frac{m_{-}^{2}}{M^{2}}-\frac{3}{16}\left(1-g_{\mathrm{s}}^{2}\right) \frac{m_{-}^{2}}{M^{2}} \frac{\pi}{\sqrt{3}}\right), \\
\delta_{H \mathrm{f}}= & \delta_{1 \mathrm{f}}=\frac{g^{2}}{16 \pi^{2}(n-4)}\left(\frac{1}{16}\left(1+\left|g_{i}\right|\right) \frac{m_{-}^{2}}{M^{2}}\right) .
\end{aligned}
$$

As a consequence of this choice of $\delta_{H}$, no $\mathrm{m}_{-}^{2}$ effects appear in the ghost sector. Notice that $\delta_{g_{i}}$ appears always accompanied by $g_{i}$ and thus only terms of zero order in $g_{i}$ are given above. Once the $\delta$ 's are substituted, $m_{-}^{2}$ effects occur only in diagrams with $\mathrm{U}$ and/or $\mathrm{H}$ external legs.

The renormalization of the propagators of the scalar fields needs some detailed discussion. The finite parts $\delta_{m}, \delta_{g_{i}}, \delta_{\mu}, \delta_{U}$ are determined in a way that the tree-level mass eigenvalues $m_{+}$and $m_{-}$remain the poles of the propagators at the one-loop level. Given that the calculation is done in terms of the non-diagonal fields $H$ and $U$, the derivation of these values involves two steps. The first step is to specify the conditions for the mass renormalization in terms of the tree-level mass eigenstates $\Omega_{ \pm}$given in eq. (4.3). Let $\Sigma_{ \pm}\left(k^{2}\right)$ be the self energies for $\Omega_{+}$ and $\Omega_{-}$and $\Sigma_{+-}\left(k^{\frac{1}{2}}\right)$ be the $\Omega_{+} \Omega_{-}$transition at one-loop. The quadratic part of the effective lagrangian is

$$
\begin{aligned}
\mathscr{L}_{\mathrm{eff}}= & -\frac{1}{2}\left(\partial_{\mu} \Omega_{+}\right)^{2}-\frac{1}{2} m_{+}^{2} \Omega_{+}^{2}-\frac{1}{2}\left(\partial_{\mu} \Omega_{-}\right)^{2}-\frac{1}{2} m_{-}^{2} \Omega_{-}^{2} \\
& +\frac{1}{2} \Sigma_{+} \Omega_{+}^{2}+\frac{1}{2} \Sigma_{-} \Omega_{-}^{2}+\Sigma_{+-} \Omega_{+} \Omega_{-}+\ldots
\end{aligned}
$$


In order to rediagonalize at the one-loop level, we need to impose

$$
\Sigma_{+-}\left(k^{2}=-m_{ \pm}^{2}\right)=0,
$$

It follows that the propagators maintain their tree-level form near the corresponding poles $m_{ \pm}$. In order for $m_{+}$and $m_{-}$to be located at the position of the poles, we should also require

$$
\Sigma_{+}\left(k^{2}=-m_{+}^{2}\right)=0 \quad \text { and } \quad \Sigma_{-}\left(k^{2}=-m_{-}^{2}\right)=0 .
$$

The second step in the mass renormalization program is to express the above conditions (eqs. (4.7), (4.8)) in terms of the self energies of the non-diagonal modes $\mathrm{H}$ and $\mathrm{U}$. Let $\Sigma_{\mathrm{H}}, \Sigma_{\mathrm{U}}, \Sigma_{\mathrm{HU}}$ be the $\mathrm{H}-\mathrm{H}, \mathrm{U}-\mathrm{U}$ and $\mathrm{H}-\mathrm{U}$ transitions, respectively. The associations between the two sets of transition amplitudes follows by subjecting $\mathscr{L}_{\text {eff }}$, eq. (4.6), to the transformation of the fields given in eq. (4.3). This leads to

$$
\Sigma_{ \pm}=\frac{1}{2}\left(\Sigma_{\mathrm{H}}+\Sigma_{\mathrm{U}} \pm 2 \frac{\left|g_{i}\right|}{g_{i}} \Sigma_{\mathrm{UH}}\right), \quad \Sigma_{+-}=\frac{1}{2}\left(\Sigma_{\mathrm{H}}-\Sigma_{\mathrm{U}}\right) .
$$

As a consequence of the mass renormalization conditions, i.e., of eqs. (4.7), (4.8) as modified by eq. (4.9), $m_{+}$and $m_{-}$are the physical masses of the two scalars.

\subsection{U-PARTICLE EFFECTS UPON THE FOUR-W AMPLITUDE}

In this section we compute the $U$ particle effects to the amplitude for four longitudinally polarized vector bosons at the tree- and one-loop levels. The vector boson scattering is shown schematically in the first of the diagrams of fig. 4 . The indices $a, b, c$ and $d$ vary from 1 to 3 and specify the isospin states of the W's. $\alpha$, $\beta, \gamma, \delta$ are the Lorentz indices and all momenta are taken to be ingoing. In the center of mass reference frame the momenta and the longitudinal polarization vectors are:

$$
\begin{aligned}
k & =\left(0,0, k_{\ell}, i k_{0}\right), \quad k^{\prime}=-\left(k_{\ell} \sin \theta, 0, k_{\ell} \cos \theta, i k_{0}\right), \\
p & =\left(0,0,-k_{\ell}, i k_{0}\right), \quad p^{\prime}=\left(k_{\ell} \sin \theta, 0, k_{\ell} \cos \theta,-i k_{0}\right), \\
\epsilon_{\alpha}(k) & =\frac{1}{M}\left(0,0, k_{0}, i k_{\ell}\right), \quad \epsilon_{\delta}\left(k^{\prime}\right)=-\frac{1}{M}\left(k_{0} \sin \theta, 0, k_{0} \cos \theta, i k_{\ell}\right), \\
\epsilon_{\beta}(p) & =\frac{1}{M}\left(0,0,-k_{0}, i k_{\ell}\right), \quad \epsilon_{\gamma}\left(p^{\prime}\right)=\frac{1}{M}\left(k_{0} \sin \theta, 0, k_{0} \cos \theta,-i k_{\ell}\right) .
\end{aligned}
$$

We work in the approximation: $M^{2} \ll s \ll m_{ \pm}^{2}$. We are interested in the leading (quadratic) terms in $s, t, u$. The computation is done in terms of the non-diagonal 

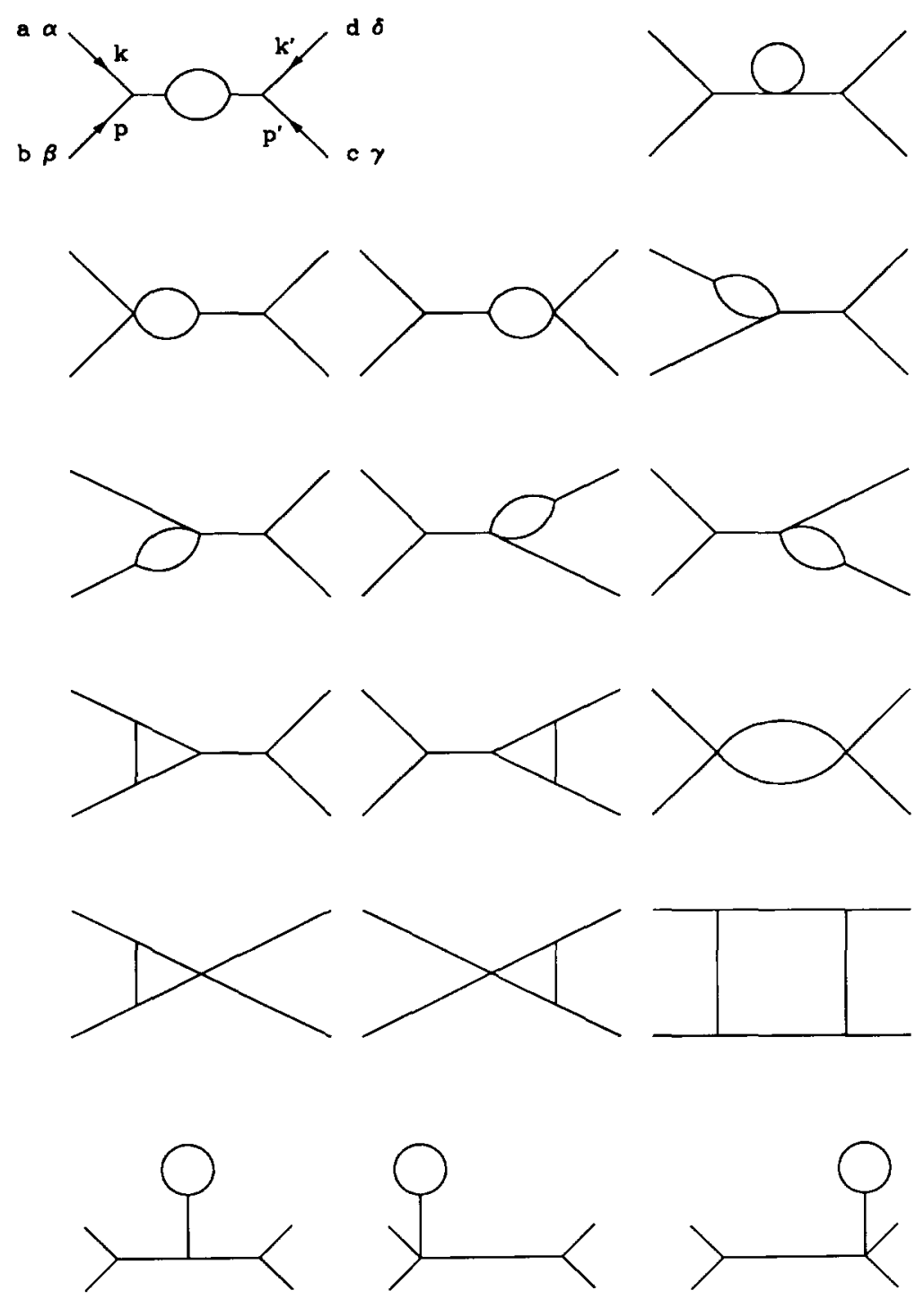

Fig. 4. Topologies for one-loop diagrams without $\mathrm{U}$ lines contributing to WW scattering.

fields $\mathrm{H}$ and $\mathrm{U}$. From them only $\mathrm{H}$ couples directly to the vector boson fields. The presence of $\mathrm{U}$ is manifested in the diagrams with $\mathrm{H}$ exchange (see eq. (4.5)). The tree-level result is

$$
\begin{aligned}
A_{\text {tree }}= & (2 \pi)^{4} i g^{2}\left[\delta_{a b} \delta_{c d} \frac{s^{2}}{4 M^{2}} \frac{1}{-s+m_{-}^{2}}\left(1-\frac{\left|g_{i}\right| m_{-}^{2}}{-s \overline{+m_{-}^{2}}}+\mathrm{O}\left(g_{i}^{2}\right)\right)\right. \\
& \left.+\delta_{a d} \delta_{c b}(s \rightarrow t)+\delta_{a c} \delta_{b d}(s \rightarrow u)\right]
\end{aligned}
$$



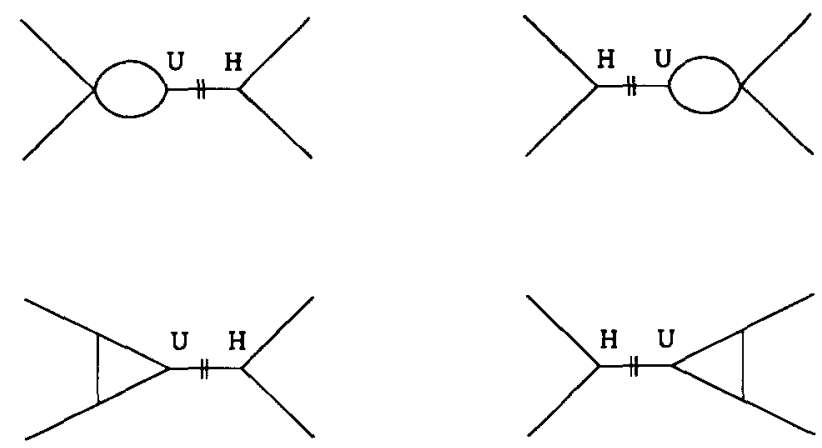

Fig. 5. Topologies for corrections with mixed UH propagator.

where $s, t, u$ are the Mandelstam parameters defined as $s=-(p+k)^{2}, t=-(p$ $\left.+p^{\prime}\right)^{2}, u=-\left(k+p^{\prime}\right)^{2}$. If $|s|,|t|,|u| \ll m_{-}^{2}$ all the above terms are suppressed by a factor $1 / m_{-}^{2}$ and may be ignored.

At the one-loop level the complete calculation of the new radiative corrections involves: (i) Diagrams without $U$ lines. The relevant topologies are shown in fig. 4. These are one-loop graphs already present in the minimal model as given in ref. [7]. The new effects arise entirely from the change of the propagator for the $\mathrm{H}$ field. (ii) Reducible three-point graphs with $U$ lines as shown in fig. 5. The $U$ lines appear in the mixing propagator $\Delta_{\mathrm{UH}}$ only. (iii) Diagrams with explicit appearance of the $U$ propagator shown in fig. 6 a. The blobs stand for the topologies of fig. $6 \mathrm{~b}$. The interrupted lines represent the mixing UH propagator.

The diagrams shown above are in the $s$-channel. The result of the $t$ - and $u$-channels is obtained by crossing, i.e., by exchanging $p \leftrightarrow k^{\prime}$ and $k \leftrightarrow k^{\prime}$, respectively. Finally, the diagrams with counterterm shown in fig. 7 must be subtracted from the above result. The crosses stand for combinations of $\delta$ 's.

For the computation of the diagrams in category (i), we may apply the calculational tricks of ref. [7]. Essential in obtaining the leading in the Higgs mass dependence is the expansion of the Higgs propagator

$$
\frac{1}{(q+p)^{2}+m^{2}}=\frac{1}{q^{2}+m^{2}}\left(1-\frac{p^{2}+2 p \cdot q}{q^{2}+m^{2}}+\frac{4(p \cdot q) p^{2}+4(p \cdot q)^{2}}{\left(q^{2}+m^{2}\right)^{2}}-\ldots\right) .
$$

Power counting arguments prescribe how many terms are kept in the expansion. In the present case, both terms $N( \pm)=\left[(q+p)^{2}+m_{ \pm}^{2}\right]^{-1}$ of the modified H propagator should be expanded accordingly. However, the power counting procedure becomes extremely complicated for terms containing both $N(+)$ and $N(-)$. To circumvent this difficulty we make the assumption of eq. (4.4) and we find the leading, linear in $g_{i}$, contributions. The result and some details of the calculation for the category (i) are given in appendix C. By inspection it is obvious that the 

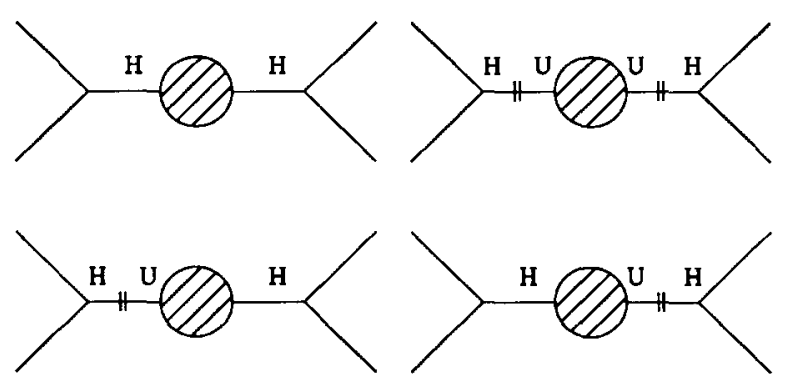

$-a-$
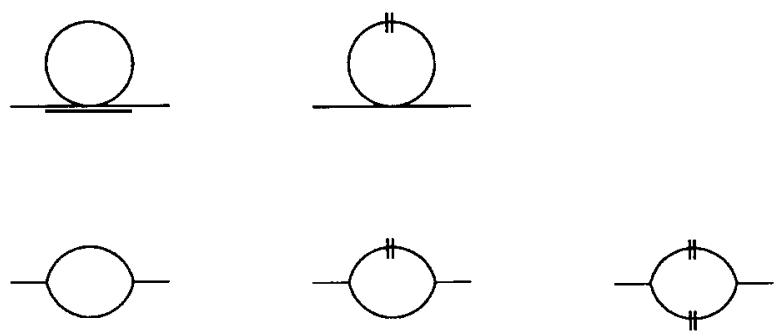

$$
-b-
$$

Fig. 6. (a) Two-point corrections with $\mathrm{U}$ lines contributing to WW scattering. (b) Topologies of two-point diagrams represented by the blob in (a).
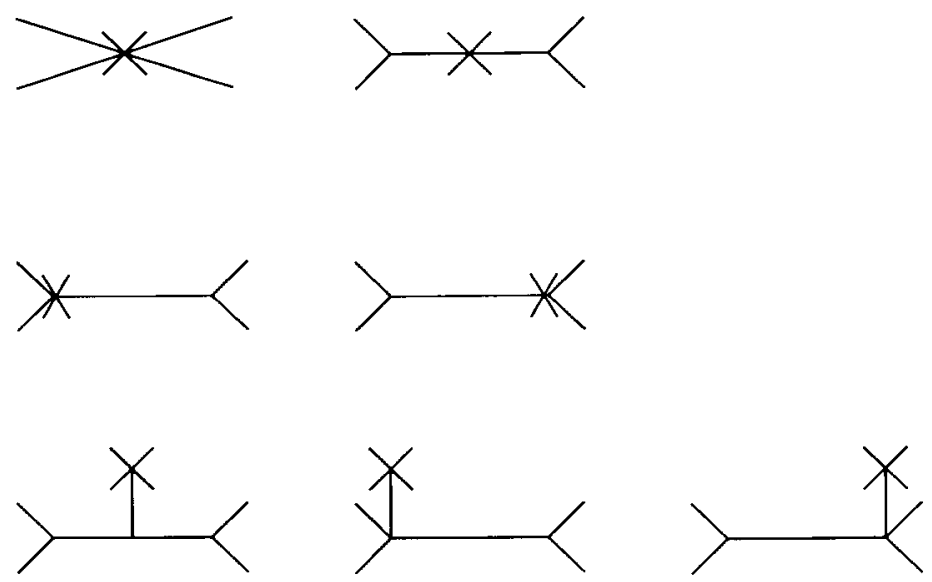

Fig. 7. Diagrams with counterterms. 
diagrams of category (ii) give terms of order $g_{i}^{2}$ and higher. Also, the diagrams with tadpoles cancel against the corresponding graphs with counterterms. Finally, the self-energy graphs of fig. 6a yield

$$
\mathrm{A}^{\mathrm{H}-\text { exchange }}=\frac{i \pi^{2}}{M^{4}} g^{4}\left|g_{i}\right|\left(\delta_{a b} \delta_{c d} \cdot \frac{3}{32} s^{2}+\delta_{a c} \delta_{b d} \cdot \frac{3}{32} u^{2}+\delta_{a d} \delta_{b c} \cdot \frac{3}{32} t^{2}\right) .
$$

The final result for the linear-in- $g_{i}$ effects to the longitudinally polarized vector boson scattering is

$$
\begin{aligned}
A_{1-\text { loop }}= & \frac{i \pi^{2}}{24 M^{4}} g^{4}\left|g_{i}\right|\left[\delta_{a b} \delta_{c d}\left(s t-\frac{1}{2} s^{2}+t^{2}\right)\right. \\
& +\delta_{a c} \delta_{b d}(s \rightarrow u, t \rightarrow s, u \rightarrow t) \\
& \left.+\delta_{a d} \delta_{b c}(s \rightarrow t, t \rightarrow u, u \rightarrow s)\right] .
\end{aligned}
$$

The complete result includes the $g_{i}$-independent terms which are precisely the effect of the minimal model given in ref. [7], with the new cutoff $m_{-}$replacing $m$.

At this point the fitting of the free parameters $g, M$ to the low-energy experimental data should be performed. In principle, this introduces new $g^{4} \ln \left(m_{-}^{2}\right)$ and $g^{4} g_{i}$ contributions. However, due to a cancellation of the quadratic terms in $s, t, u$ at the tree level, no new effects are added to the leading order result given above. This may still be expressed in another way: Due to the aforementioned cancellation at the tree level, it can be shown in that the four-W amplitude at the leading order depends only on $\delta_{m}, \delta_{g_{i}}$ and $\delta_{\mu}$ (i.e., the renormalization of the masses of the scalars), while the rest of the $\delta$ 's cancel among themselves. The surviving $\delta$ 's appear suppressed by a factor $1 / m_{ \pm}^{2}$ in the diagrams with counterterms. Consequently, terms growing like $\ln \left(m_{ \pm}^{2}\right)$ in the $\delta$ 's are irrelevant to the result for the one-loop amplitude at leading order in the scalar masses. We conclude that that the total new effects are given in eq. (4.12) with the experimental values substituted for the parameters.

\subsection{THE $\beta$ PARAMETER}

In this section we use the result for the corrections to the $\mathrm{W}_{\mathrm{L}} \mathrm{W}_{\mathrm{L}}$ amplitude to derive the new contributions to the Lehmann $\beta$ parameter. First, let us review the definition of this variable. Based on unitarity and analiticity arguments (dispersion relations method) [15], the low-energy result (linear in $s, t, u$ ) for the $\mathrm{W}_{\mathrm{L}} \mathrm{W}_{\mathrm{L}}$ scattering amplitude may be extended to a higher range in terms of two undetermined parameters $\beta_{1,2}$ :

$$
A\left(\mathrm{~W}_{\mathrm{L}}^{a} \mathrm{~W}_{\mathrm{L}}^{b} \rightarrow \mathrm{W}_{\mathrm{L}}^{c} \mathrm{~W}_{\mathrm{L}}^{d}\right)=\delta_{a b} \delta_{c d} F(s, t, u)+\delta_{a c} \delta_{b d} F(u, t, s)+\delta_{a d} \delta_{b c} F(t, s, u),
$$


with

$$
\begin{aligned}
F(s, t, u)= & \frac{s}{v^{2}}-\frac{1}{96 \pi^{2} v^{4}}\left\{3 s^{2}\left[\ln (s)-\beta_{1}\right]+t(t-u)\left[\ln (t)-\beta_{2}\right]\right. \\
& \left.+u(u-t)\left[\ln (u)-\beta_{2}\right]\right\}
\end{aligned}
$$

where $v=2 \mathrm{M} / \mathrm{g}=250 \mathrm{GeV}$ is the vacuum expectation value of the Higgs doublet. The corresponding pion result is given by substituting $v \rightarrow f_{\pi}$. The partial waves $t_{l}^{I}(s)$ may then be calculated and used to specify the phase shifts from $t_{l}^{I}=\left(\cot \delta_{l}^{I}\right.$ $-i)^{-1}$. Finally the effective range approximation is taken by expanding $\cot \delta$ at $s=0$. The resonance is located at the position where $\cot \delta$ vanishes. In particular, the $I=J=1$ partial wave exhibits a pole at

$$
s=\frac{288 \pi^{2} v^{2}}{9 \beta-1}
$$

Notice that the position of the pole depends on $\beta \equiv \beta_{2}-\beta_{1}$ whose value is undetermined. Lehmann attempts to derive it from a first order perturbative result.

Based on the reasonable success of this method in pion physics, the parameter $\beta$ was calculated in ref. [14] from the one-loop amplitude of $\mathrm{W}_{\mathrm{L}} \mathrm{W}_{\mathrm{L}}$ scattering. In a similar manner eq. (4.12) yields the following $U$-dependent contributions:

$$
\begin{gathered}
\beta_{1}(U)=-\left|g_{i}\right|, \quad \beta_{2}(U)=\left|g_{i}\right| \\
\beta \equiv \beta_{2}-\beta_{1}=2\left|g_{i}\right| .
\end{gathered}
$$

By adding the $g_{i}$-independent terms, we have the complete result for model B:

$$
\beta=-2-9\left(\frac{\pi}{\sqrt{3}}-2\right)+2\left|g_{i}\right|=-0.32+2\left|g_{i}\right| \text {. }
$$

Given that we have assumed $\left|g_{i}\right| \ll 1$ (eq. (4.4)), the parameter maintains its negative value and thus, according to eq. (4.15), no resonance appears at any energy.

\subsection{SUMMARY AND DISCUSSION OF THE RESULTS}

We have computed the $U$ particle effects upon the $W_{L} W_{L}$ scattering amplitude at the tree and one-loop levels. We have considered model B which describes a scalar $U$ particle that develops a vacuum expectation value and thus mixes with the traditional Higgs scalar at the tree level. We have assumed a heavy and strongly 
interacting scalar sector where both mass scales are infinitely larger than the other scales in the problem. We have also assumed that the values of the scalar masses are close, the measure of their difference being given by the parameter $g_{i}$ which is taken to be small. The strength of the coupling between the scalars remains strong despite the size of $g_{i}$. The computation was done in the form of an expansion in this parameter and the leading order (linear in $g_{i}$ ) terms were found. For $g_{i} \leqslant 0.1$ the new corrections are roughly of the order of $10 \%$ and our perturbative result gives a reasonable estimate of the behaviour of the model. Furthermore, this expansion allows us to extract the results of the minimal model: They are simply described by the terms of zero order in $g_{i}$. At the tree level we found negligible effects (see eq. (4.10)) suppressed by a factor $1 / m_{-}^{2}$. The non-zero result at the one-loop level (see eq. (4.12)) illustrates the arbitrariness of the heavy-Higgs theory, a fact already demonstrated in terms of model A [7]. It may be interesting to notice that, while in model $A$ the new contributions originate exclusively in self-energy corrections of the $\mathrm{H}$ field, this is not the case for the model considered here. Also, let us point out that eq. (4.12) does not contain $g_{i} \ln \left(m_{-}^{2}\right)$ terms and thus, the leading (logarithmic) corrections with respect to the Higgs mass are U-independent.

The result for the four-W amplitude was then used to derive the value of the parameter $\beta$ within the framework of partial wave analysis. The virtue of this parameter is its independence of $\ln \left(m_{-}^{2}\right)$ effects. In this way it gives a definite prediction for the position of a resonance in the $I=1$ channel (analogous to the $\rho$ resonance in $\pi \pi$ scattering). The new corrections are found to be positive. However, due to the assumed small value of $g_{i}$, they can not counteract the negative value obtained by the minimal model. Thus the overall result for $\beta$ is negative indicating that there is no resonance at any energy. It is interesting to notice that our result, along with other known examples of a singlet particle extension of the standard model $[14,16]$, tends to move the value of $\beta$ toward the positive range where resonances start appearing. One might wonder whether this positivity of the $U$ particle effects on $\beta$ holds true for all similarly extended models. Due to technical difficulties, we were unable to show this for the complete (non-perturbative with respect to $g_{i}$ ) result for model B. Nonetheless, if this were the case, a large difference between the scalar masses might push the value of $\beta$ in the positive range. In this sense one might ask whether, through the additional $\mathrm{U}$-particles, we might have an indication of a spectrum of resonances in the $\mathrm{TeV}$ regime.

We would like to thank Professor M. Veltman for suggesting this problem, for valuable discussions and for the introduction to SCHOONSCHIP. We also wish to thank R. Akhoury for discussions. We would like to thank the high energy theory group at Brookhaven National laboratory for its warm hospitality and the use of its facilities. 


\section{Appendix A}

For the two-loop calculation of sect. 3 the methods developed in ref. [5] are used. In this appendix we review a few basic formulae. By differentiating or using partial fractions all two-loop integrals can be reduced to the basic one:

$$
\begin{aligned}
\left\langle m_{0} m_{0}\left|m_{1}\right| m_{2}\right\rangle \equiv & \int \mathrm{d}^{n} q \mathrm{~d}^{n} p \frac{1}{\left(p^{2}+m_{0}^{2}\right)^{2}} \frac{1}{q^{2}+m_{1}^{2}} \frac{1}{(p+q)^{2}+m_{2}^{2}} \\
= & \pi^{4}\left\{-\frac{2}{\epsilon^{2}}+\frac{1}{\epsilon}\left[1-2 \ln \left(m_{0}^{2}\right)\right]-\frac{1}{2}-\frac{1}{12} \pi^{2}-\ln m_{0}^{2}\right. \\
& \left.-\ln ^{2} m_{0}^{2}-f(a, b)\right\},
\end{aligned}
$$

where $a=m_{1}^{2} / m_{0}^{2}, b=m_{2}^{2} / m_{0}^{2}$ and

$$
\begin{aligned}
f(a, b)= & -\frac{1}{2} \ln ^{2}(a)-\operatorname{Sp}\left(\frac{a-b}{a}\right) \\
& +\left(\frac{a+b-1}{2 \sqrt{1-2(a+b)+(a-b)^{2}}}-\frac{1}{2}\right) \\
& \times\left[\operatorname{Sp}\left(\frac{b-a}{x_{1}}\right)-\operatorname{Sp}\left(\frac{a-b}{1-x_{1}}\right)-\operatorname{Sp}\left(1-\frac{1}{x_{1}}\right)+\operatorname{Sp}\left(-\frac{x_{1}}{1-x_{1}}\right)\right] \\
& -\left(\frac{a+b-1}{2 \sqrt{1-2(a+b)+(a-b)^{2}}}+\frac{1}{2}\right) \\
& \times\left[\operatorname{Sp}\left(\frac{b-a}{x_{2}}\right)-\operatorname{Sp}\left(\frac{a-b}{1-x_{2}}\right)-\operatorname{Sp}\left(1-\frac{1}{x_{2}}\right)+\operatorname{Sp}\left(-\frac{x_{2}}{1-x_{2}}\right)\right],
\end{aligned}
$$

with

$$
x_{1,2}=\frac{1}{2}\left[1+b-a \pm \sqrt{1-2(a+b)+(a-b)^{2}}\right]
$$

The Spence function or dilogarithm is defined as

$$
\mathrm{Sp}(x) \equiv-\int_{0}^{1} \frac{\mathrm{d} t}{t} \ln (1-x t) .
$$

We give below a few details concerning the generation of the terms with Clausen functions which are the signature of the ambiguity between linear and 
non-linear models. If $a=b>\frac{1}{4}$ the argument of the Spence functions is complex of unit measure. The series expansion of a Spence function for $|z|=1$ is

$$
\operatorname{Sp}(z)=\sum_{k=1}^{\infty} \frac{\exp (i k \phi)}{k^{2}}=\sum_{k=1}^{\infty} \frac{\cos -k \phi}{k^{2}}+i \sum_{k=1}^{\infty} \frac{\sin k \phi}{k^{2}}
$$

where

$$
\mathrm{Cl}(\phi)=\sum_{k=1}^{\infty} \frac{\sin k \phi}{k^{2}}, \quad \phi=\arctan \frac{\sqrt{4 a-1}}{1-2 a}
$$

For $a=1$ there appears terms with the factor $\mathrm{Cl}(\pi / 3)$ which is the signature of the ambiguous effects.

In case of momentum dependent integrals (relevant to the $\mathrm{W}$ mass shift) we may find the leading dependence in the Higgs mass $m$, as follows. Given that we work in the heavy-Higgs limit, we may proceed by expanding the corresponding integrals about the position of zero external momentum. For this, we expand the internal propagator

$$
\frac{1}{(q+p)^{2}+M^{2}}=\frac{1}{q^{2}+M^{2}}\left(1-\frac{p^{2}+2 p \cdot q}{q^{2}+M^{2}}+\frac{4(p \cdot q) p^{2}+4(p \cdot q)^{2}}{\left(q^{2}+M^{2}\right)^{2}}-\ldots\right)
$$

The expansion allows us to use the generic form of eq. (A.1) for the calculation of the momentum-dependent two-loop integrals. Also, the $p_{\mu}$-independent terms are immediately singled out. From the remaining series only a limited number of terms given $m^{2}$ contribution. The dependence of the various integrals on $m$ may be determined from the following rule: Find the dimensionality of the integral in question by means of naive power counting. This specifies the highest power of the heavy mass that can appear in the result.

\section{Appendix B}

In this appendix we give the Feynman rules for the SU(2) gauge theory with the extended scalar sector given by model B (see eq. (4.1)). The additional singlet particle $U$ develops a vacuum expectation value and mixes at the lagrangian level with the original scalar field $\mathrm{H}$. Due to the $\mathrm{U}-\mathrm{H}$ mixing of zeroth order in $g$, the complete tree-level propagators are obtained by summing up an infinite series.

$$
\text { H propagator: } \Delta_{\mathrm{H}}=\frac{\frac{1}{2}}{(2 \pi)^{4} i}\left(\frac{y}{k^{2}+m_{+}^{2}-i \epsilon}+\frac{\underline{1-y}}{k^{2}+m_{-}^{2}-i \epsilon}\right) \text {, }
$$




$$
\begin{aligned}
\text { U propagator: } \Delta_{\mathrm{U}} & =\frac{\frac{1}{2}}{(2 \pi)^{4} i}\left(\frac{\left.\frac{1-y}{k^{2}+m_{+}^{2}-i \epsilon}+\frac{y}{k^{2}+m_{-}^{2}-i \epsilon}\right),}{\text { U-H propagator: } \Delta_{\mathrm{UH}}}=\frac{\frac{1}{2}}{(2 \pi)^{4} i} y^{\prime}\left(\frac{1}{k^{2}+m_{+}^{2}-i \epsilon}-\frac{1}{k^{2}+m_{-}^{2}-i \epsilon}\right),\right.
\end{aligned}
$$

where

$$
y=\frac{m_{+}^{2}-m_{u}^{2}}{m_{+}^{2}-m_{-}^{2}} \quad \text { and } \quad y^{\prime}=\frac{g_{i} m_{\mathrm{H}}^{2}}{m_{+}^{2}-m_{-}^{2}} .
$$

The mass eigenstates are

$$
\Omega_{ \pm}=\frac{1}{\sqrt{\left(g_{i} m_{\mathrm{H}}^{2}\right)^{2}+\left(m_{+}^{2}-m_{\mathrm{H}}^{2}\right)^{2}}}\left[ \pm g_{i} m_{\mathrm{H}}^{2} H+\left(m_{+}^{2}-m_{\mathrm{H}}^{2}\right) U\right]
$$

corresponding to the mass eigenvalues

$$
m_{ \pm}^{2}=\frac{1}{2}\left[m_{\mathrm{H}}^{2}+m_{\mathrm{u}}^{2} \pm \sqrt{\left(m_{\mathrm{H}}^{2}-m_{\mathrm{u}}^{2}\right)^{2} \pm 4\left(g_{i} m_{\mathrm{H}}^{2}\right)^{2}}\right] .
$$

In sect. 4 we make a particular choice of the parameters given in eq. (4.2), i.e., $m_{\mathrm{H}}^{2}=m_{\mathrm{u}}^{2}=m^{2}$. As a consequence, the Feynman rules become (all momenta are taken to be ingoing)

W propagator: $\quad \Delta_{\mu \nu}^{a b}(\mathrm{~W})=\frac{\delta_{a b} \delta_{\mu \nu}}{k^{2}+M^{2}-i \epsilon}$,

Higgs-ghost propagator: $\quad \Delta^{a b}(\phi)=\frac{\delta_{a b}}{k^{2}+M^{2}-i \epsilon}$,

F-P ghost propagator: $\quad \Delta^{a b}(\chi)=\frac{\delta_{a b}}{k^{2}+M^{2}-i \epsilon}$,

H propagator:

$$
\Delta_{\mathrm{H}}=\frac{\frac{1}{2}}{(2 \pi)^{4} i}\left(\frac{1}{k^{2}+m_{-}^{2}-i \epsilon}+\frac{1}{k^{2}+m_{+}^{2}-i \epsilon}\right),
$$

U propagator:

$$
\Delta_{\mathrm{U}}=\frac{\frac{1}{2}}{(2 \pi)^{4} i}\left(\frac{1}{k^{2}+m_{-}^{2}-i \epsilon}+\frac{1}{k^{2}+m_{+}^{2}-i \epsilon}\right),
$$

$\mathrm{U}-\mathrm{H}$ propagator:

$$
\Delta_{\mathrm{UH}}=\frac{\frac{1}{2}}{(2 \pi)^{4} i} \frac{\left|g_{i}\right|}{g_{i}}\left(\frac{1}{k^{2}+m_{+}^{2}-i \epsilon}-\frac{1}{k^{2}+m_{-}^{2}-i \epsilon}\right),
$$


Vertices:

$$
\begin{aligned}
& W_{\alpha}^{a}(k) W_{\beta}^{b}(p) W_{\gamma}^{c}(q): \quad-i \epsilon_{a b c}\left[\delta_{\alpha \gamma}(k-q)_{\beta}+\delta_{\beta \gamma}(q-p)_{\alpha}+\delta_{\alpha \beta}(p-k)_{\gamma}\right], \\
& W_{\alpha}^{a} W_{\beta}^{b} W_{\gamma}^{c} W_{\delta}^{d}: \quad-g^{2}\left[\epsilon_{e d c} \epsilon_{e b a}\left(2 \delta_{\alpha \gamma} \delta_{\beta \delta}-\delta_{\alpha \delta} \delta_{\beta \gamma}-\delta_{\alpha \beta} \delta_{\gamma \delta}\right)\right. \\
& \left.+\epsilon_{e d b} \epsilon_{e c a}\left(2 \delta_{\alpha \beta} \delta_{\gamma \delta}-\delta_{\alpha \delta} \delta_{\beta \gamma}-\delta_{\alpha \gamma} \delta_{\beta \delta}\right)\right] \\
& W_{\alpha}^{a}(k) \phi^{b}(p) \phi^{c}(q): \quad \frac{1}{2} i g \epsilon_{a b c}(p-q)_{\alpha}, \\
& W_{\alpha}^{a}(k) H \phi^{b}(p): \quad \quad \frac{1}{2} i g \delta_{a b}(p-q)_{\alpha}, \\
& W_{\alpha}^{a} W_{\beta}^{b} H: \quad-\frac{1}{2} g M \delta_{a b} \delta_{\alpha \beta}, \\
& \chi^{* a}(q) \chi^{b}(p) W_{\alpha}^{c}(k): \quad i g \epsilon_{a b c} p_{\alpha}, \\
& \chi^{* a} \chi^{b} H: \quad-\frac{1}{2} m g \delta_{a b}, \\
& \chi^{* a} \chi^{b} \phi^{c}: \quad \quad \frac{1}{2} g M \epsilon_{a b c}, \\
& W_{\alpha}^{a} W_{\beta}^{b} \phi^{c} \phi^{d}: \quad-\frac{1}{2} g^{2} \delta_{\alpha \beta} \delta_{a b} \delta_{c d}, \\
& W_{\alpha}^{a} W_{\beta}^{b} H^{2}: \quad \quad \frac{1}{2} g^{2} \delta_{\alpha \beta} \delta_{a b}, \\
& \phi^{a} \phi^{b} H \\
& -g \frac{m^{2}}{2 M} \delta_{a b} \\
& H^{3} \text { : } \\
& -g \frac{3 m^{2}}{2 M} \\
& H U^{2} \text { : } \\
& -\frac{1}{2} g_{i} g_{\mathrm{s}} g \frac{m^{2}}{M}, \\
& H^{2} U \\
& -\frac{1}{2} g_{i} g \frac{m^{2}}{M} \\
& \phi^{a} \phi^{b} U: \quad-\frac{1}{2} g_{i} g \frac{m^{2}}{M} \delta_{a b}, \\
& U^{3}: \quad-\frac{3}{2} g_{\mathrm{s}} g \frac{m^{2}}{M}, \\
& \phi^{a} \phi^{b} H H: \quad-\frac{1}{4} g^{2} \frac{m^{2}}{M^{2}} \delta_{a b},
\end{aligned}
$$




$$
\begin{array}{ll}
\phi^{a} \phi^{b} \phi^{c} \phi^{d}: & -g^{2} \frac{m^{2}}{4 M^{2}}\left(\delta_{a b} \delta_{c d}+\delta_{a c} \delta_{b d}+\delta_{a d} \delta_{c a}\right), \\
H^{4}: & -g^{2} \frac{3 m^{2}}{4 M^{2}}, \\
\phi^{a} \phi^{b} U U: & -\frac{1}{4} g_{i} g^{2} g_{\mathrm{s}} \frac{m^{2}}{M^{2}} \delta_{a b}, \\
\text { HHUU: } & -\frac{1}{4} g_{i} g^{2} g_{\mathrm{s}} \frac{m^{2}}{M^{2}}, \\
\text { UUUU: } & -\frac{3}{4} g^{2} g_{\mathrm{s}}^{2} \frac{m^{2}}{M^{2}}
\end{array}
$$

\section{Appendix C}

In this appendix we describe a special trick which simplified considerably the calculation of the one-loop graphs without $U$ lines for the four-vector boson amplitude. All calculations were done using the algebraic manipulation program SCHOONSCHIP. The terms with linear dependence in $g_{i}$ arise from: (i) The $m^{2}$ factors in the vertices, since $m^{2}=m_{-}^{2}\left(1+\left|g_{i}\right|+\mathrm{O}\left(g_{\mathrm{i}}^{2}\right)\right)$ and (ii) The $g_{i}$-dependent term of the propagator $\Delta_{\mathrm{H}}$ as given in eq. (4.5). Notice that this term may be obtained by a differentiation of the $g_{i}$-independent term of $\Delta_{\mathrm{H}}$ as follows:

$$
\left.\frac{\mathrm{d}}{\mathrm{d} a}\left(\frac{1}{k^{2}+a m_{-}^{2}}\right)\right|_{a=1}=\frac{-m_{-}^{2}}{\left(k^{2}+m_{-}^{2}\right)^{2}} .
$$

Based on this remark, it is easy to see that the result may be obtained by dublicating the steps involved in the calculation with the minimal model as was done in ref. [7]. One has merely to rescale the $m_{-}^{2}$ factors in the $\mathrm{H}$ propagator (and not in the vertices) by $a m_{-}^{2}$. In this way we are able to discern in the final result between the $m_{-}$factors coming from the vertices and the ones coming from the propagators and differentiate accordingly. The final result for the contribution of the diagrams without $\mathrm{U}$ lines is

$$
\begin{aligned}
A= & \frac{i \pi^{2}}{M^{4}} g^{4} g_{i}\left[\delta_{a b} \delta_{c d}\left(\frac{s t}{24}-\frac{11 s^{2}}{96}+\frac{t^{2}}{24}\right)\right. \\
& +\delta_{a c} \delta_{b d}(s \rightarrow u, t \rightarrow s, u \rightarrow t) \\
& \left.+\delta_{a d} \delta_{b c}(s \rightarrow t, t \rightarrow u, u \rightarrow s)\right] .
\end{aligned}
$$




\section{References}

[1] M. Veltman, Acta Phys. Polon. B8 (1977) 475

[2] B.W. Lee, C. Quigg and H.B. Thacker, Phys. Rev. D16 (1977) 1519

[3] M.S. Chanowitz and M.K. Gaillard, Nucl. Phys. B261 (1985) 379

[4] T. Appelquist and C. Bernard, Phys. Rev. D22 (1980) 200; D23 (1981) 424;

A. Longhitano, Phys. Rev. D22 (1980) 1166; Nucl. Phys. B188 (1981) 118;

R. Akhoury and Y.P. Yao, Phys. Rev. D25 (1982) 3361

[5] J. van der Bij and M. Veltman, Nucl. Phys. B231 (1984) 205

[6] J. van der Bij, Nucl. Phys. B248 (1984) 141

[7] M. Veltman and F. Ynduráin, Nucl. Phys. B325 (1989) 1

[8] D. Ross and M. Veltman, Nucl. Phys. B95 (1975) 135

[9] M. Veltman, The Higgs System, Lectures Lorentz Institute (Leiden)

[10] S. Dawson and S. Willenbrock, Phys. Rev. Lett. 62 (1989) 1232;

G. Passarino, Nucl. Phys. B343 (1990) 31;

H. Veltman, Phys. Rev. D43 (1991) 2236

[11] P. Hung and H. Thacker, Phys. Rev. D31 (1985) 2866;

R. Casalbuoni, S. de Curtis, D. Dominici and R. Gatto, Phys. Lett. B155 (1985) 95; Nucl. Phys. B282 (1987) 235;

P. Chiappetta and S. Narison, Phys. Lett. B198 (1987) 421;

P. Hung, T.N. Pham and T. Truong, Phys. Rev. Lett. 59 (1987) 2251;

R. Rosenfeld and J.L. Rosner, Phys. Rev. D38 (1988) 1530

[12] R. Casalbuoni, D. Dominici and F. Feruglio, R. Gatto, Phys. Lett. B200 (1988) 495;

M.S. Chanowitz and M. Golden, Phys. Rev. Lett. 61 (1988) 1053;

R. Rosenfeld, Mod. Phys. Lett. A4 (1989) 1999;

A. Dobado, M.J. Herrero and T. Truong, Phys. Lett. B235 (1990) 129;

S. Dawson, G. Valencia, Nucl. Phys. B352 (1991) 27

[13] J.M. Cornwall, D.N. Levin and G. Tiktopoulos, Phys. Rev. D10 (1974) 1145;

G.J. Gounaris, R. Kogerler and H. Neufeld, Phys. Rev. D34 (1986) 3257;

Y.P. Yuan and C.P. Yao, Phys. Rev. D38 (1988) 2237;

G. Valencia and S. Willenbrock, Phys. Rev. D42 (1990) 843;

H. Veltman, Phys. Rev. D41 (1990) 2294

[14] H. Veltman and M. Veltman, Acta Phys. Pol. B22 (1991) 669

[15] H. Lehmann, Acta Phys. Austr. Suppl. XI (1973) 139

[16] J.J. van der $\mathrm{Bij}$ and $\mathrm{H}$. Veltman, DESY preprint 91-118

[17] A. Hill and J.J. van der Bij, Phys. Rev. D36 (1987) 3463

[18] M. Green and M. Veltman, Nucl. Phys. B169 (1980) 137

[19] T. Appelquist and J. Carazzone, Phys. Rev. D11 (1975) 2856 